\title{
Quadrilateral elements for the solution of elasto-plastic finite strain problems
}

\author{
José M. A. César de Sá*,t,‡, Pedro M. A. Areias ${ }^{\S}$ and Renato M. Natal Jorgeף \\ Instituto de Engenharia Mecânica (IDMEC), Faculdade de Engenharia da Universidade do Porto, \\ Rua dos Bragas, 4050-123 Porto, Portugal
}

\begin{abstract}
SUMMARY
In this paper two plane strain quadrilateral elements with two and four variables, are proposed. These elements are applied to the analysis of finite strain elasto-plastic problems. The elements are based on the enhanced strain and B-bar methodologies and possess a stabilizing term. The pressure and dilatation fields are assumed to be constant in each element's domain and the deformation gradient is enriched with additional variables, as in the enhanced strain methodology. The formulation is deduced from a four-field functional, based on the imposition of two constraints: annulment of the enhanced part of the deformation gradient and the relation between the assumed dilatation and the deformation gradient determinant. The discretized form of equilibrium is presented, and the analytical linearization is deduced, to ensure the asymptotically quadratic rate of convergence in the Newton-Raphson method. The proposed formulation for the enhanced terms is carried out in the isoparametric domain and does not need the usually adopted procedure of evaluating the Jacobian matrix in the centre of the element. The elements are very effective for the particular class of problems analysed and do not present any locking or instability tendencies, as illustrated by various representative examples. Copyright (c) 2001 John Wiley \& Sons, Ltd.
\end{abstract}

KEY WORDS: finite strains; elasto-plasticity; enhanced strains; finite elements

\section{INTRODUCTION}

The deficiencies of the standard low-order plane strain elements, related to locking behaviour, are visible in many problems, such as in the analysis of near-incompressible situations (including elasto-plastic problems as investigated by Nagtegaal et al. [1]) and in bending-dominated situations.

\footnotetext{
*Correspondence to: José César de Sá, Department of Mechanical Engineering, University of Porto, Rua dos Bragas, 4099 Porto Cedex, Portugal

†E-mail: cesarsa@fe.up.pt

¥Associate Professor

$\S$ Ph.D. Student

TAssistant Professor
}

Contract/grant sponsor: PRAXIS; contract/grant number: XXI/BD/18533/98

Copyright (c) 2001 John Wiley \& Sons, Ltd. 
However, due to their simplicity, this class of elements is attractive for non-linear analyses, and therefore, some improvements have been carried out over the years to eliminate that undesirable behaviour. The use of higher order elements (quadratic and cubic) usually avoids these difficulties, particularly when based on a mixed formulation [2]. Nevertheless, they are more sensitive to mesh distortion [3] and the contact calculations are more difficult. Under these conditions, properly formulated low-order elements are suitable to a wide range of applications.

For the linear elastic case, there are some reliable formulations as those described in References [4-6], therefore, the non-linear case is addressed in this paper. On the contrary, for the non-linear case, the main difficulty is the trade-off between stability and accuracy. In fact, it is the presence of instabilities (the so-called hourglass patterns) in several important cases of compression and tension that has somehow compromised promising formulations (for example the enhanced strain element of Simo and Armero [7]).

The enhanced strain method, theoretically justified for non-linear problems by Simo and Armero [7], consists in the enrichment of the deformation gradient with additional variables. It is noticeable that, to satisfy the patch test, it is necessary to perform an ad hoc modification of the enhanced term (classified as a trick by Lautersztajn and Samuelsson in Reference [8]), based on the central evaluation of the Jacobian co-ordinate.

As an extension of the standard element, the enhanced strain method is very convenient for the computational implementation of elasto-plastic material models, because the algorithmic treatment is unchanged. On the contrary, the hybrid formulations (such as presented by Pian and Sumihara [5]) and two-field $u-p$ formulations (described, for example, by Brink and Stein [9]) usually demand specific treatment.

Despite the generally good results obtained with the enhanced strain elements (for elastic problems see Reference [10]), they present some imperfections such as increased mesh distortion sensitivity and marked instabilities that appear in numerous practical problems [11, 3, 12]; even in the original work, Simo and Armero [7] recognize the presence of spurious modes in situations of high dilatational deformations. Owing to this drawback, several authors proposed modifications to the original work. Simo et al. [3] introduced a five-point integration rule, as opposed to the original four-point rule, with the argument that the original rule under-integrates the enhanced strain element. Notwithstanding, the instabilities are only mildly attenuated (as illustrated by Glaser and Armero [12]). Additionally, the resulting element loses flexibility and computational efficiency.

The work developed by Korelc and Wriggers [13] over a single element's instability led to the introduction of additional orthogonality conditions and to the modification of the interpolation for the deformation-gradient-enhanced part. These improvements attenuated the compression instabilities. However, the discrete strain-displacement operators lose their original sparsity.

Subsequently, Glaser and Armero [12] alleged lack of objectivity in the previous proposal [13] and introduced a correction based on a further evaluation of the deformation gradient in the element's centre. Additionally, to tackle the tension instability, a stabilization term was added to the original functional and five-point or nine-point integration rules were tested. Despite its effectiveness in controlling the instabilities, this last contribution increases the analytical complexity of the element, particularly in the evaluation of the tangent stiffness, and further increases the computational cost due to the additional integration points. 
The attenuation of the mesh distortion sensitivity has also drawn attention of some researchers. For this purpose, the truncated Taylor expansion of the shape functions proposed by Wriggers and Hueck [14] and Korelc and Wriggers [15] appears to be an effective way of increasing the element's robustness.

It is important to note that the enhanced strain methodology is not the only strategy developed with the aim of improving the results of the standard element. There are some worth noting alternative strategies. The mean-dilatation technique of Nagtegaal et al. [1], and the closely related B-bar technique presented by Hughes [16] and further extended by Simo et al. [17] and Moran et al. [18], are appropriate to the analysis of nearly incompressible problems. These techniques are based on the assumption of an independent interpolation of the dilatation field. The derivations are supported by a three-field functional [17]. The B-bar technique can be related, under certain conditions, to the selective integration technique (as discussed by Hughes [16]). There is also an ad hoc B-bar procedure to avoid the shear locking (as discussed by Zhu and Cescotto [19]). A particularly important issue is the linearization results of the B-bar elements (addressed by Simo et al. [17] and Moran et al. [18]) that will be detailed in this paper.

Although the results obtained with the B-bar formulation are frequently good, certain authors [3] have noticed a diffuse response in strain localization problems.

Another approach, advocated by various authors, is the reduced integration technique with a stabilizing term (as detailed in References [20-23]) which is very efficient and avoids locking tendencies. However, this technique presents some drawbacks. In Reference [21], Reese et al. proposes a stabilization technique based on an 'equivalent parallelogram' that can induce a dependence between the results and the selected load increments (see Reference [21]), which can be undesirable.

Besides this limitation, the single-point integration can be inadequate for elasto-plastic problems (as stated by Zhu and Cescotto [19]).

There are also some important contributions regarding the mixed $u-p$ methods, with pressure and displacement variables (a review is presented by Brink and Stein [9]). These methods are usually based on two-field functionals (as adopted by Bathe [24]) or three-field functionals (as in the recent paper by Crisfield [25]). This last formulation is closely related to the B-bar presented by Simo et al. [17].

Recently, some mixed/enhanced strain formulations were introduced, namely with the contributions of Piltner and Taylor [26] with added internal stress variables and Pantuso and Bathe [2] who included dilatation and pressure variables. In another context, Taylor [27] applied a mixed/enhanced formulation to triangular elements. These mixed/enhanced formulations typically imply an increase in the number of variables and therefore, are computationally more expensive than the usual enhanced strain elements.

Inspired by these last two works [2, 27], the authors of this paper propose two enhanced strain/B-bar elements with assumed dilatation and enhanced distortional part of the deformation gradient. These two elements extend a previous work carried out by César de Sá and Natal Jorge [4]. The fundamental difference between the two elements is the number of additional variables: two or four. Therefore, the number of variables does not exceed the number of variables present in the standard enhanced strain element.

The spurious mode control is carried out through a stabilization term included in the original functional that imposes the annulment of the enhanced part of the deformation gradient. 
The variational formulation as well as the discretization are fully detailed. The linearization calculations, necessary to obtain an asymptotically quadratic rate in the Newton-Raphson algorithm are also described.

The proposed formulation is tested by several representative numerical examples, with elastic and elasto-plastic materials, covering a variety of situations. All the examples show the effectiveness of the two newly proposed elements and the absence of spurious modes.

It is worth noting that, during the course of submission of this work, a paper describing an apparently similar enhanced strain/B-bar element formulation has been published by Armero [28]. However, as it will become clear, although there are some coincident points between the two formulations, they present some differences.

\section{INCOMPRESSIBILITY AND LOCKING}

A known deformation of a body denoted as $\mathscr{B}$ is said to be incompressible if any region of the body has a constant volume.

This condition can be stated by the equation $\operatorname{div} \mathbf{v}(X)=0$, which can be obtained through the conservation of mass equation, imposing $\rho_{0}=\rho$ where $\rho$ is the mass density in a given configuration and $\rho_{0}$ is the mass density in the reference configuration.

According to this definition, a given deformation is incompressible when the spatial divergence of the velocity is null in any point $X$ of $\mathscr{B}$.

Denoting $J=\operatorname{det}[\mathbf{F}], \mathbf{F}$ being the deformation gradient, it is possible to restate the incompressibility condition as

$$
J(X)=1
$$

An equally important case, due to its practical importance, is that of near-incompressible case. The elasto-plastic materials, for isochoric plastic flows, are frequently near-incompressible, since the dilatation is purely elastic.

When numerical quadrature is used, the incompressibility condition is verified only in each integration point. The numerical integration induces a relaxation in the incompressibility constraint.

The locking behaviour, in the incompressible situation, of the standard four-node element is related to the absence of the two hourglass deformation modes [29, 30, 4]. In fact, as referred by de Souza Neto et al. [31] there is an inherent inability in the interpolation functions in the correct representation of isochoric displacement fields.

For a four node square element, César de Sá et al. [4, 29, 30] have shown the relation between the incompressibility constraint, imposed at the four integration points, and the absence of three deformation modes: the dilatation mode and the two hourglass modes. The lack of hourglass deformation is responsible for the volumetric locking.

That analysis, carried out for the linear case, is further extended here, through the annulment condition of the velocity spatial divergence:

$$
\operatorname{div} \mathbf{v}=0 \Rightarrow \frac{\partial v_{i}}{\partial x_{i}}=0
$$

The discretized form of the velocity field, in each element, is stated as $v_{i}\left(\xi_{1}, \xi_{2}\right)=N^{k}\left(\xi_{1}, \xi_{2}\right) v_{i}^{k}$, where $N^{k}$ is the shape function of node $k$. The nodal velocity variables are denoted as $v_{i}^{k}$. The co-ordinates $\xi_{1}$ and $\xi_{2}$ are denominated local co-ordinates of the element. 
According to this discretized form, it is possible to write, for the referred particular case,

$$
\frac{\partial v_{i}}{\partial x_{i}}\left(\xi_{1}, \xi_{2}\right)=\frac{\partial N^{k}}{\partial \xi_{i}}\left(\xi_{1}, \xi_{2}\right) v_{i}^{k}
$$

Imposing condition (2) in the standard four Gauss integration points (e.g. Reference [24]) whose local co-ordinates are denoted as $\xi_{1}^{k}, \xi_{2}^{k}, k=1, \ldots, 4$, it is possible to write

$$
\left\{\begin{array}{l}
\operatorname{div} \mathbf{v}\left(\xi_{1}^{1}, \xi_{2}^{1}\right) \\
\operatorname{div} \mathbf{v}\left(\xi_{1}^{2}, \xi_{2}^{2}\right) \\
\operatorname{div} \mathbf{v}\left(\xi_{1}^{3}, \xi_{2}^{3}\right) \\
\operatorname{div} \mathbf{v}\left(\xi_{1}^{4}, \xi_{2}^{4}\right)
\end{array}\right\}=[\mathbf{Q}]\{\mathbf{v}\}
$$

where [Q] is the matrix described in References [4, 30]; making use of the same notation, this matrix is defined for a square element in the usual local co-ordinates, i.e. with side size equal to 2 , as

$$
[\mathbf{Q}]=\left[\begin{array}{llllllll}
-a_{1} & -a_{1} & a_{1} & -a_{2} & a_{2} & a_{2} & -a_{2} & a_{1} \\
-a_{2} & -a_{1} & a_{2} & -a_{2} & a_{1} & a_{2} & -a_{1} & a_{1} \\
-a_{1} & -a_{2} & a_{1} & -a_{1} & a_{2} & a_{1} & -a_{2} & a_{2} \\
-a_{2} & -a_{2} & a_{2} & -a_{1} & a_{1} & a_{1} & -a_{1} & a_{2}
\end{array}\right]
$$

where $a_{1}=0.25\left(1+a_{0}\right), a_{2}=0.25\left(1-a_{0}\right)$ and $a_{0}=1 / \sqrt{3}$.

The conclusions of César de Sá et al., presented in References [4, 29, 30] keep their validity in the present finite strain case. The point-wise incompressibility constraint for a standard square element is stated as

$$
[\mathbf{Q}]\{\mathbf{v}\}=\{0\}
$$

The hourglass modes and the dilatation mode do not belong to the space of solutions of nodal velocity vectors $\{\mathbf{v}\}$ that satisfy $(6)$.

If a reduced/selective integration is used, the incompressibility constraint is imposed in the element's central point, and in the mean dilatation technique, the dilatation is calculated as an average of its values at the four integration points.

For the selective integration case, César de Sá et al. [4, 29, 30] have shown, based on condition (6), that the hourglass modes, previously absent, are present in this case.

The $[\mathbf{Q}]$ matrix, corresponding to the selective integration case, can be written as

$$
[\mathbf{Q}]=\frac{1}{4}\left[\begin{array}{lllllllll}
-1 & -1 & 1 & -1 & 1 & 1 & -1 & 1
\end{array}\right]
$$

In the mean-dilatation technique, the incompressibility constraint is verified in a global fashion, since the equation $v_{e}=V_{e}$ is satisfied, which states the coincidence between the material and spatial volumes in each element for any isochoric deformation.

As it will become clear in this paper, the mean-dilatation method is strictly equivalent to the selective integration method, if the volume ratio is calculated exactly.

Kinematically, it is possible to locally decompose the deformation gradient in a distortional term and a dilatational term (as in Reference [31]). This type of decomposition can be specially useful when there is the intent of defining distinct interpolations for these two terms, as it is the case in this paper. 
The kinematic decomposition can be stated as

$$
\mathbf{F}=\hat{\mathbf{F}} \mathbf{F}^{V}
$$

In agreement with Equation (8), the deformation gradient can be decomposed in a distortional term, such as $\hat{\mathbf{F}}$ : $\operatorname{det}[\hat{\mathbf{F}}]=1$ and a volumetric term $\mathbf{F}^{V}=\mathbf{I} J^{1 / 3}$ (where $\mathbf{I}$ denotes the identity tensor), which can also be denominated as dilatation term because it corresponds to a pure dilatation deformation.

In harmony with the previous remarks, the distortional term can be calculated through the following relation:

$$
\hat{\mathbf{F}}=J^{-1 / 3} \mathbf{F}
$$

The dilatation term will be considered as 'assumed' for the elements proposed in this paper.

\section{A FOUR-FIELD FUNCTIONAL}

The analysis of near-incompressible problems is frequently based on functionals with two or three independent fields. In the two-field case, a penalty parameter is present, related to the bulk modulus [24], and the pressure $p$ and the spatial position $\mathbf{x}$ are the assumed independent fields. In the three-field case, the pressure is a Lagrange multiplier and the independent fields are: the pressure itself $p$, the dilatation $\theta$ and the spatial position $\mathbf{x}[17,32]$. In this paper, a four-field functional is proposed. This functional is an extension of the three-field functional presented in Reference [17].

As usual, a particle of the body $\mathscr{B}$, observed in a given reference configuration, denoted as $\mathbb{B}$, is given by its position in that configuration, $\mathbf{X}$.

It is assumed that the reference configuration $\mathbb{B}$ is subjected to a conservative body force whose density is denoted by $\mathbf{B}$. The surface forces are also included, identified by the nominal stress vector $\overline{\mathbf{T}}$ defined in a subset of the reference configuration boundary, $\Gamma_{t} \subset \partial \mathbb{B}$.

In a subset of the boundary, $\Gamma_{x} \subset \partial \mathbb{B}$, at least one component of the spatial position is prescribed. It is also assumed that $\Gamma_{t} \cap \Gamma_{x}=\emptyset$.

A potential function associated with these conservative forces is written as

$$
\Pi_{\text {ext }}(\mathbf{x})=-\int_{\mathbb{B}} \rho_{0} \mathbf{B} \cdot \mathbf{x} \mathrm{d} V-\int_{\Gamma_{t}} \overline{\mathbf{T}} \cdot \mathbf{x} \mathrm{d} V
$$

where $\rho_{0}$ is the mass density in the reference configuration.

The special class of materials considered in this paper is characterized by a stored energy function $W$, which can be related to the free energy function [33]. This function depends on the right Cauchy-Green deformation tensor $\mathbf{C}=\mathbf{F}^{\mathrm{T}} \mathbf{F}$.

The first Piola-Kirchhoff stress tensor, denoted as $\mathbf{P}$, can be determined by differentiating $W$ with respect to $\mathbf{F}$, i.e. $\mathbf{P}=\partial_{\mathbf{F}} W$, and the Kirchhoff stress tensor is related to $\mathbf{P}$ by the equation $\tau=\mathbf{P F}^{\mathrm{T}}$.

The elastic potential energy is calculated by the following expression:

$$
\Pi_{\text {int }}(\mathbf{x})=\int_{\mathbb{B}} W(\mathbf{C}) \mathrm{d} V
$$


The total potential is the sum of the internal and external potentials:

$$
\Pi(\mathbf{x})=\Pi_{\text {int }}(\mathbf{x})+\Pi_{\text {ext }}(\mathbf{x})
$$

From the imposition of the stationarity condition for potential (12), it is possible to obtain the local form of the momentum equations and the described boundary conditions.

The key idea in the following developments is the pre-established independence of the following fields:

- Spatial position: $\mathbf{x}(\mathbf{X})$

- Dilatation: $\theta(\mathbf{X})$

- Deformation gradient: $\mathbf{F}(\mathbf{X})$

As this independence is assumed, it turns out to be necessary to impose local (i.e. valid for each $\mathbf{X} \in \mathbb{B}$ ) constraints to relate the fields. These constraints are added to the original form of the functional (12).

The considered local constraints can be written as

$$
\begin{aligned}
& \theta=\operatorname{det}[\mathbf{F}] \\
& \mathbf{F}=\nabla_{0} \mathbf{x} \Leftrightarrow\left(\mathbf{F}-\nabla_{0} \mathbf{x}\right):\left(\mathbf{F}-\nabla_{0} \mathbf{x}\right)=0
\end{aligned}
$$

The scalar components of $\nabla_{0} \mathbf{x}$ are written as $\nabla_{0} x_{i j}=\partial x_{i} / \partial X_{j}$.

The second constraint, in its scalar form, allows the use of a single parameter (or a Lagrange multiplier), in contrast with the tensorial form, introduced by Simo and Armero [7] that makes use of a second order tensorial Lagrange multiplier which is the first Piola-Kirchhoff stress tensor.

In agreement with this particular form for the constraints, the resulting functional possesses two scalar constraints.

It is noticeable that, according to the multiplicative decomposition of $\mathbf{F}$, defined by Equation (8), it is possible to introduce an assumed deformation gradient, with an assumed dilatation field, making use of the following notation:

$$
\tilde{\mathbf{F}}=\theta^{1 / 3} \hat{\mathbf{F}}
$$

where $\hat{\mathbf{F}}$ is the distortional part of $\mathbf{F}$ :

$$
\hat{\mathbf{F}}=J^{-1 / 3} \mathbf{F}
$$

and $J=\operatorname{det}[\mathbf{F}]$.

According to these developments and noting the definition $J=\operatorname{det}[\mathbf{F}]$, the local constraints can be rewritten as

$$
\begin{aligned}
& \theta=\operatorname{det}[\mathbf{F}] \\
&\left(\sqrt[3]{\frac{J}{\theta}} \tilde{\mathbf{F}}-\nabla_{0} \mathbf{x}\right):\left(\sqrt[3]{\frac{J}{\theta}} \tilde{\mathbf{F}}-\nabla_{0} \mathbf{x}\right)=0
\end{aligned}
$$

With this notation, the first constraint represents the relation $J=\theta$ and the second one is equivalent to $\nabla_{0} \mathbf{x}=\mathbf{F}$. 
The second constraint will be imposed by means of a penalty parameter and the first one by a scalar Lagrange multiplier which can be straightforwardly related to the pressure field, $p$. If $r$ represents the penalty parameter, then, a four-field functional is written as

$$
\Pi(\mathbf{x}, \mathbf{F}, p, \theta)=\int_{\mathbb{B}}[W\left(\tilde{\mathbf{F}}^{\mathrm{T}} \tilde{\mathbf{F}}\right)+\underbrace{\frac{r}{2}\left(\mathbf{F}-\nabla_{0} \mathbf{x}\right):\left(\mathbf{F}-\nabla_{0} \mathbf{x}\right)}_{S}+p(J-\theta)] \mathrm{d} V+\Pi_{\text {ext }}(\mathbf{x})
$$

where $\mathbf{F}=\sqrt[3]{(J / \theta)} \tilde{\mathbf{F}}$.

A practical advantage of this functional, in comparison with the modified three-field principle introduced in Reference [7], is the imposition of the deformation-gradient constraint without the necessity of explicitly assuming the first Piola-Kirchhoff stress tensor. In the case of the formulation proposed by Armero [28], the first Piola-Kirchhoff stress tensor must be assumed as well. This author proposes a five-field functional, to subsequently use an orthogonality condition, so that the assumed first Piola-Kirchhoff tensor, corresponding to a Lagrange multiplier, is absent from the final equilibrium equations. The present form is distinct from the one proposed by Armero [28].

The criterion for the determination of the penalty parameter will be addressed later.

The inclusion of the term identified as $S$, related to the imposition of the constraint (13b), acts as a stabilizing term, where the $r$ parameter allows the adjustment of the relation $\mathbf{F}=\nabla_{0} \mathbf{x}$.

This term avoids the necessity of assumptions described by Simo and Armero [7] about the constant Piola-Kirchhoff stress field.

It is noticeable that in the classical enhanced strain formulation this constraint is generally not verified, as it is assumed that there is an orthogonality between the multiplier tensor and the constraint $\mathbf{F}=\nabla_{0} \mathbf{x}$ (see the assumptions established by Simo and Armero in Reference [7]).

The stationarity condition of the proposed functional (17) gives the momentum equation, the boundary conditions, and if $r \rightarrow \infty$, the local imposed constraints.

Imposing the annulment of the variation of functional (17), it can be stated that

$$
\begin{array}{r}
\int_{\mathbb{B}}\left[\sqrt[3]{\frac{\theta}{J}} \tilde{\mathbf{P}}+r\left(\mathbf{F}-\nabla_{0} \mathbf{x}\right)-\frac{1}{3}\left(\frac{\theta}{J}\right)^{-1 / 3}(\tilde{\mathbf{P}}: \mathbf{F}) \mathbf{F}^{-\mathrm{T}}+p J \mathbf{F}^{-\mathrm{T}}\right]: \delta \mathbf{F} \mathrm{d} V=0 \\
\int_{\mathbb{B}}\left[\frac{1}{3 J}\left(\frac{\theta}{J}\right)^{-2 / 3} \tilde{\mathbf{P}}: \mathbf{F}-p\right] \delta \theta \mathrm{d} V=0 \\
\int_{\mathbb{B}}(J-\theta) \delta p \mathrm{~d} V=0 \\
\int_{\mathbb{B}}-r\left(\mathbf{F}-\nabla_{0} \mathbf{x}\right): \nabla_{0} \delta \mathbf{x} \mathrm{d} V+\delta \Pi_{\text {ext }}(\mathbf{x})=0
\end{array}
$$

where $\tilde{\mathbf{P}}=\partial_{\tilde{\mathbf{F}}} W$ is the first Piola-Kirchhoff stress tensor calculated with previously introduced assumed deformation gradient. 
These equations were calculated using the definition $J=\operatorname{det}[\mathbf{F}]$ and the following equation:

$$
\delta J=J \mathbf{F}^{-\mathrm{T}}: \delta \mathbf{F}
$$

From Equation (18c), it is possible to write the following relation:

$$
J=\operatorname{det}[\mathbf{F}]=\theta
$$

Isolating the pressure, $p$, from Equation (18b), the result is

$$
p=\frac{1}{3 J} \tilde{\mathbf{P}}: \mathbf{F}=\frac{1}{3 J} \operatorname{tr} \tilde{\tau}
$$

where $\tilde{\tau}=\tilde{\mathbf{P}} \mathbf{F}^{\mathrm{T}}$.

Equation (18a) and the previous relations allow the following equation for the first Piola-Kirchhoff stress tensor:

$$
\tilde{\mathbf{P}}=-r\left(\mathbf{F}-\nabla_{0} \mathbf{x}\right)
$$

This equation is somewhat predictable as it relates the introduced penalty parameter with the Lagrange multiplier used in the work of Simo and Armero [7] to impose the relation $\mathbf{F}-\nabla_{0} \mathbf{x}$. The stress tensor $\tilde{\mathbf{P}}$ is the cited Lagrange multiplier, assumed to be constant in the work of Simo and Armero [7].

Clearly, if $r \rightarrow \infty$, the local constraint $\mathbf{F}=\nabla_{0} \mathbf{x}$ should be satisfied.

The substitution of relation (22) in Equation (18d) gives the virtual work principle in the material form. Making use of the notation $\nabla_{0} \delta \mathbf{x}=\nabla \delta \mathbf{x F}$ and noting the property $\tilde{\mathbf{P}}:(\nabla \mathbf{x F})=$ $\tilde{\mathbf{P}} \mathbf{F}^{\mathrm{T}}: \nabla \mathbf{x}$, it is possible to write the virtual work principle in the spatial form

$$
\int_{\mathbb{B}} \tau: \nabla \delta \mathbf{x} \mathrm{d} V=\int_{\mathbb{B}} \rho_{0} \mathbf{B} \cdot \delta \mathbf{x} \mathrm{d} V+\int_{\Gamma_{t}} \overline{\mathbf{T}} \cdot \delta \mathbf{x} \mathrm{d} a
$$

which is a weak form of the local momentum equation.

The purpose of this exposition was to clarify the introduction functional (17), and to show that it includes the local momentum equations, boundary conditions and constraint equations. It is noticeable that there has not been any discussion about appropriate particular forms for the dilatation $\theta$, pressure $p$, and deformation gradient $\mathbf{F}$. This will be the objective of the following sections.

\section{ASSUMED FIELDS}

The general concepts presented in Section 3 will be used to develop two plane strain elements with assumed dilatation and pressure and 2 or 4 internal variables, respectively.

These elements have an additional stabilization term, that corresponds to the imposed constraint with the penalty parameter $r$. It will become apparent that the stabilization term is extremely simple when compared with others (for example References [20, 21]), specially in the linearized form. 


\subsection{Assumed deformation gradient}

The particular form of $\mathbf{F}$ was not imposed in the previous discussion. The notation $J=\operatorname{det}[\mathbf{F}]$ was established, and, as $\mathbf{F}$ will be assumed, implies that, generally, $J \neq \operatorname{det}\left[\nabla_{0} \mathbf{x}\right]$.

The denomination for the dilatation, $\theta$, is here a synonym of assumed dilatation, since a different interpolation will be used for this field. Also, as computationally $1 / r$ is never null, there is a numerical difference between $\nabla_{0} \mathbf{x}$ and $\mathbf{F}$.

In a similar fashion, as locally $J \neq \theta$, then in general $\mathbf{F} \neq \tilde{\mathbf{F}}$, which requires some precaution with the nomenclature used, particularly in the definition of the spatial tensors.

According to the already presented functional, the central idea consists in re-parameterizing the deformation gradient with the assumed dilatation, through the following equation:

$$
\tilde{\mathbf{F}}=\sqrt[3]{\frac{\theta}{J}}\left(\nabla_{0} \mathbf{x}+\mathbf{A}\right)
$$

The term $\mathbf{A}$ is calculated by an interpolation and can be viewed as an enhancement to the deformation gradient.

Comparing the equation (24) with the one originally proposed in Reference [7], it can be stated that the dilatation is assumed and the distortional part of the deformation gradient is enhanced. Therefore, this formulation is equivalent to a projection (using the nomenclature used by Simo et al. [17]) of the deformation gradient of an enhanced strain element. Equation (24) has been introduced by the authors in Reference [34].

It is noticeable that, for Equation (24), it is implied that the expression $\sqrt[3]{(1 / J)}\left(\nabla_{0} \mathbf{x}+\mathbf{A}\right)$ is the distortional part of the deformation gradient. The dilatation, $\theta$, is imposed in an independent fashion of the particular form of $\sqrt[3]{(1 / J)}\left(\nabla_{0} \mathbf{x}+\mathbf{A}\right)$. The important comments about the nonintersection of the spaces spanned by $\nabla_{0} \mathbf{x}$ and $\mathbf{A}$ carried out by Simo and Armero [7], remain valid in this particular projected form.

Equation (24), in the absence of $\mathbf{A}$, is formally equivalent to that presented by Simo et al. [17].

A hypothetical alternative form for Equation (24), would consist in writing $\tilde{\mathbf{F}}=\sqrt[3]{\left(\theta^{\prime} / J^{\prime}\right)}$ $\nabla_{0} \mathbf{x}+\mathbf{A}^{\prime}$, with now $J^{\prime}=\operatorname{det}\left[\nabla_{0} \mathbf{x}\right]$. However, this type of decomposition, which was explored by the authors, introduces some complexities in the final form of the discretized equations and was, therefore, abandoned.

The examples presented in this paper obey to the plane strain hypothesis. For that case, the $3 \times 3$ deformation gradient is written as follows:

$$
\mathbf{F}_{3 \times 3}=\left[\begin{array}{ccc}
F_{11} & F_{12} & 0 \\
F_{21} & F_{22} & 0 \\
0 & 0 & 1
\end{array}\right]
$$

from which a $2 \times 2$ matrix may be defined as

$$
\mathbf{F}_{2 \times 2}=\left[\begin{array}{ll}
F_{11} & F_{12} \\
F_{21} & F_{22}
\end{array}\right]
$$

It is clear that $\tilde{\mathbf{F}}$ should have a form similar to (25), which assures the plane strain hypothesis. 
Therefore, the discussed tensors $\nabla_{0} \mathbf{x}, \mathbf{F}$ and $\tilde{\mathbf{F}}$, are, under plane strain conditions, two dimensional tensors. Hence, decomposition (24) is written as

$$
\tilde{\mathbf{F}}=\sqrt{\frac{\theta}{J}}\left(\nabla_{0} \mathbf{x}+\mathbf{A}\right)
$$

where the square root follows from the property $\operatorname{det}\left[c \mathbf{F}_{n \times n}\right]=c^{n} \operatorname{det}\left[\mathbf{F}_{n \times n}\right]$ where $c$ is a scalar and $n$ is the dimension of $\mathbf{F}$.

This particular form appears to be different from the one proposed by Armero [28].

Nevertheless, the original form (24) is used in the remaining discussions, without loss of generality.

\subsection{Stationarity condition}

By inserting Equation (24) in functional (17), it is possible to write it as

$$
\Pi(\mathbf{x}, \mathbf{A}, p, \theta)=\int_{\mathbb{B}}\left[W(\theta, \mathbf{x}, \mathbf{A})+\frac{r}{2} \mathbf{A}: \mathbf{A}+p(J-\theta)\right] \mathrm{d} V+\Pi_{\text {ext }}(\mathbf{x})
$$

Given this final form for (17), the form adopted for the stabilization term in the initial functional is now clear. The penalty parameter, $r$, allows the adjustment of the local constraint $\mathbf{A}=\mathbf{0}$.

Before imposing the stationarity conditions to (28) we will establish some relations and notations that will be useful.

The notation used here for the enhanced deformation gradient is

$$
\mathbf{F}=\nabla_{0} \mathbf{x}+\mathbf{A}
$$

where $\mathbf{F}$ is analog to the one proposed by Simo and Armero [7] (and distinct from the assumed deformation gradient $\tilde{\mathbf{F}}$ ).

The first variation of $J$ can be written through the enhanced deformation gradient, according to the following equation:

$$
\delta J=J \delta \mathbf{F}: \mathbf{F}^{-\mathrm{T}}
$$

Two divergence operators may be introduced as

$$
\begin{aligned}
& \operatorname{div} \delta \mathbf{x}=\nabla_{0} \delta \mathbf{x}: \mathbf{F}^{-\mathrm{T}}=(\nabla \delta \mathbf{x F}): \mathbf{F}^{-\mathrm{T}} \\
& \underline{\operatorname{div}} \delta \mathbf{a}=\delta \mathbf{A}: \mathbf{F}^{-\mathrm{T}}=(\delta \mathbf{a F}): \mathbf{F}^{-\mathrm{T}}
\end{aligned}
$$

The notation $\underline{\operatorname{div}} \delta \mathbf{a}$ is a convenient notation, and does not correspond to a truly divergence operator.

Inserting these definitions in Equation (30), the following relation is obtained:

$$
\delta J=J \operatorname{div} \delta \mathbf{x}+J \underline{\operatorname{div}} \delta \mathbf{a}
$$

From these equations, it is possible to write the first variation of the assumed deformation gradient, $\tilde{\mathbf{F}}$ as:

$$
\delta \tilde{\mathbf{F}}=\left[\frac{\delta \theta}{3 \theta}-\frac{1}{3}(\operatorname{div} \delta \mathbf{x}+\underline{\operatorname{div}} \delta \mathbf{a})\right] \tilde{\mathbf{F}}+\sqrt[3]{\frac{\theta}{J}} \nabla_{0} \delta \mathbf{x}+\sqrt[3]{\frac{\theta}{J}} \delta \mathbf{A}
$$


The variation of the stored energy function, $W$, can be calculated resorting to the first Piola-Kirchhoff stress tensor, $\tilde{\mathbf{P}}$, as

$$
\delta W=\partial_{\tilde{\mathbf{F}}} W: \delta \tilde{\mathbf{F}}=\tilde{\mathbf{P}}: \delta \tilde{\mathbf{F}}
$$

The already alluded spatial tensors $\nabla \delta \mathbf{x}$ and $\delta \mathbf{a}$ can be written as

$$
\begin{aligned}
\nabla_{0} \delta \mathbf{x} & =\nabla \delta \mathbf{x F} \\
\delta \mathbf{A} & =\delta \mathbf{a F}
\end{aligned}
$$

It is important to note that these two Equations (36), (37) are notations and not applications of the chain rule. This is also true to the average spatial divergence operators that will be introduced in Section 4.3.

From these conclusions and the stationarity condition of functional (28), it is possible to present the following set of equations:

$$
\begin{aligned}
\int_{\mathbb{B}}\left[\sqrt[3]{\frac{\theta}{J}} \tilde{\mathbf{P}}:\left(\delta \mathbf{A}+\nabla_{0} \delta \mathbf{x}\right)+p J(\underline{\operatorname{div}} \delta \mathbf{a}+\operatorname{div} \delta \mathbf{x})\right. \\
\left.+\frac{1}{3} \tilde{\mathbf{P}} \tilde{\mathbf{F}}(\underline{\operatorname{div}} \delta \mathbf{a}+\operatorname{div} \delta \mathbf{x})+r \mathbf{A}: \delta \mathbf{A}\right] \mathrm{d} V+\delta \Pi_{\mathrm{ext}}=0 \\
\int_{\mathbb{B}}\left(\frac{1}{3 \theta} \tilde{\mathbf{P}}: \tilde{\mathbf{F}} \delta \theta-p \delta \theta\right) \mathrm{d} V=0 \\
\int_{\mathbb{B}}(J-\theta) \delta p \mathrm{~d} V=0
\end{aligned}
$$

It can be observed that Equation (38a) is a B-bar extension of the enhanced strain formulation.

Equations (38) are the formal support of the discretized equilibrium equations. The particular forms of the pressure and dilatation fields, will now be discussed.

\subsection{Dilatation and assumed pressure}

The discretization of the fields $p$ and $\theta$ in each element's reference domain (denoted as $\mathbb{B}$, which is the notation of reference configuration of a body) can be made compatible or incompatible with the adjacent elements.

In particular, these fields can be assumed constant in each element. Using this approximation, there is a particularly simple relation between $\theta$ and $J$.

In detail, using Equation (38c), the dilatation is written as the following average:

$$
\theta=\frac{1}{V} \int_{\mathbb{B}} J \mathrm{~d} V=\frac{v}{V}
$$

in which $v$ is the current volume of the element. 
This dilatation is generally distinct from the one obtained by the displacement field alone. This can be stated as the following inequality:

$$
v \neq v_{x}=\int_{\mathbb{B}} \operatorname{det}\left[\nabla_{0} \mathbf{x}\right] \mathrm{d} V
$$

$v_{x}$ being the current volume obtained from the displacement field alone.

This inequality may raise some questions about the physical significance of the volume $v$. The particular form adopted to the term $\mathbf{A}$ has a direct influence on the relation between the displacement field and the deformation gradient $\mathbf{F}$.

When $\mathbf{F}$ is calculated through the incompatible mode method (briefly described in the Reference [7]), the displacement field is clearly defined. However, for the more general enhanced strain formulation it may not be possible to identify the displacement field from the enhanced deformation gradient.

This conclusion led the authors to investigate a methodology that avoids the contribution of $\mathbf{A}$ in (39) to the assumed dilatation.

In the case where $\mathbf{A} \equiv \mathbf{0}$, the B-bar quadrilateral element can be formulated on a meandilatation basis (as in Reference [17]) or on the selective integration of the dilatation (as in Reference [18]). The two strategies are, in fact, analytically equivalent as it will be shown.

The small differences in the results, frequently obtained (in the linear case César de Sá and Natal Jorge [4] noticed slight differences), are due to integration errors present in the mean dilatation method.

It will also be shown that the reduced integration strategy leads to an exact value of the dilatation.

For the mean dilatation technique $[1,17]$, the dilatation is calculated as follows:

$$
\theta=\frac{1}{V} \int_{\mathbb{B}} \operatorname{det}\left[\nabla_{0} \mathbf{x}\right] \mathrm{d} V=v_{x} / V
$$

If a Gauss integration rule is used to evaluate Equation (41), and as the expression $\operatorname{det}\left[\nabla_{0} \mathbf{x}\right]$ is generally a rational function of the element's co-ordinates, the volume $v_{x}$ determined by (39) is generally different from the exact deformed volume of the element. If the thickness is assumed constant, the exact deformed volume can be easily determined.

For a constant thickness field, denoted as $h$, the exact volume of a standard deformed element can be stated as

$$
v_{x}=\frac{h}{2}\left[\left(x_{3}-x_{1}\right)\left(y_{4}-y_{2}\right)+\left(x_{2}-x_{4}\right)\left(y_{3}-y_{1}\right)\right]
$$

$x_{i}$ and $y_{i}$ being the current global co-ordinates of a node $i$.

The mean dilatation of the element is calculated, according to this equation, as follows:

$$
\theta=\frac{\left[\left(x_{3}-x_{1}\right)\left(y_{4}-y_{2}\right)+\left(x_{2}-x_{4}\right)\left(y_{3}-y_{1}\right)\right]}{\left[\left(X_{3}-X_{1}\right)\left(Y_{4}-Y_{2}\right)+\left(X_{2}-X_{4}\right)\left(Y_{3}-Y_{1}\right)\right]}
$$

where $X_{i}$ and $Y_{i}$ are the global reference co-ordinates of a node $i$.

Equation (43) is coincident with that obtained with a central point integration of $\operatorname{det}\left[\nabla_{0} \mathbf{x}\right]$. This statement can be verified using the shape function derivatives at the element's centre (as deduced by Flanagan and Belytschko in Reference [35]) and calculating, from those expressions, the gradient $\nabla_{0} \mathbf{x}$. 
Conclusively, the four-point numerical integration of $\operatorname{det}\left[\nabla_{0} \mathbf{x}\right]$ is generally inaccurate, and the reduced integration of the same expression is clearly exact.

From Equation (38b) it is possible to isolate the assumed pressure, $p$, which can be related to the other fields through the following equation:

$$
p=\frac{1}{3 V \theta} \int_{\mathbb{B}} \tilde{\mathbf{P}}: \tilde{\mathbf{F}} \mathrm{d} V
$$

This equation allows the interpretation that the assumed pressure $p$ is a mean element pressure.

The definition of dilatation (39) allows the possibility to define mean spatial divergence operators (in agreement with the notation introduced by Simo et al. [17]), using the dilatation variation, $\delta \theta$, and the following property:

$$
\delta J=J(\operatorname{div} \delta \mathbf{x}+\underline{\operatorname{div}} \delta \mathbf{a})
$$

The dilatation variation can be written as

$$
\delta \theta=\frac{1}{V} \int_{\mathbb{B}} \delta J \mathrm{~d} V=\frac{1}{V} \int_{\mathbb{B}} J \operatorname{div} \delta \mathbf{x} \mathrm{d} V+\frac{1}{V} \int_{\mathbb{B}} J \underline{\operatorname{div}} \delta \mathbf{a} \mathrm{d} V
$$

From Equations (46) it is possible to relate the fields $\delta \theta, \delta \mathbf{x}$ and $\delta \mathbf{a}$ and eliminate $\delta \theta$ from Equations (38).

According to this conclusion, the dilatation variation is written as

$$
\delta \theta=\theta \overline{\operatorname{div} \delta \mathbf{x}}+\theta \overline{\operatorname{div} \delta \mathbf{a}}
$$

where the terms

$$
\begin{aligned}
& \overline{\operatorname{div} \delta \mathbf{x}}=\frac{1}{\theta V} \int_{\mathbb{B}} J \operatorname{div} \delta \mathbf{x} \mathrm{d} V \\
& \overline{\operatorname{div} \delta \mathbf{a}}=\frac{1}{\theta V} \int_{\mathbb{B}} J \underline{\operatorname{div}} \delta \mathbf{a} \mathrm{d} V
\end{aligned}
$$

are definitions of the average divergence operators. In fact, these are weighted averages of $\operatorname{div} \delta \mathbf{x}$ and $\underline{\operatorname{div}} \delta \mathbf{a}$, which is in an extension of the referred work developed by Simo et al.

In fact, Equation (48a) is similar to that presented in the work of Simo et al. [17] and Equation (48b) is an extension of the concept presented by that author for the enhanced term A introduced here. This extension is supported by the notation previously introduced for the term $\underline{\operatorname{div}} \delta \mathbf{a}$.

\subsection{Equilibrium equations}

It is now possible to simplify the form of Equation (38a), using relations (48) and some further relations.

After some analytical calculations, the following equation can be written

$$
\int_{\mathbb{B}} p J(\operatorname{div} \delta \mathbf{x}+\underline{\operatorname{div}} \delta \mathbf{a}) \mathrm{d} V=\int_{\mathbb{B}} \frac{1}{3} \tilde{\mathbf{P}}: \tilde{\mathbf{F}}(\overline{\operatorname{div} \delta \mathbf{x}}+\overline{\operatorname{div} \delta \mathbf{a}}) \mathrm{d} V
$$


The nomenclature for the assumed pressure is introduced as

$$
\bar{p}=\frac{1}{3} \tilde{\mathbf{P}}: \tilde{\mathbf{F}}
$$

therefore, it is possible to write

$$
\int_{\mathbb{B}}\left[\sqrt[3]{\frac{\theta}{J}} \tilde{\mathbf{P}}:\left(\delta \mathbf{A}+\nabla_{0} \delta \mathbf{x}\right)+r \mathbf{A}: \delta \mathbf{A}+\bar{p}(\overline{\operatorname{div} \delta \mathbf{a}}-\underline{\operatorname{div}} \delta \mathbf{a}+\overline{\operatorname{div} \delta \mathbf{x}}-\operatorname{div} \delta \mathbf{x})\right] \mathrm{d} V+\delta \Pi_{\text {ext }}=0
$$

Further, the term $\tilde{\mathbf{F}}=\sqrt[3]{(\theta / J)} \mathbf{F}$ can be isolated from Equation (51).

In this paper the equilibrium is written in a spatial setting, with the use of the Kirchhoff stress tensor $\tilde{\boldsymbol{\tau}}=\tilde{\mathbf{P}} \tilde{\mathbf{F}}^{\mathrm{T}}$.

By separating, in (51), the terms that depend on $\delta \mathbf{a}$ from the terms that depend on $\delta \mathbf{x}$ and transforming the results to the spatial configuration, the following set of equations can be written

$$
\begin{gathered}
\int_{\mathbb{B}} \tilde{\tau}: \nabla \delta \mathbf{x}+\bar{p}(\overline{\operatorname{div} \delta \mathbf{x}}-\operatorname{div} \delta \mathbf{x}) \mathrm{d} V+\delta \Pi_{\mathrm{ext}}=0 \\
\int_{\mathbb{B}} \tilde{\tau}: \delta \mathbf{a}+\bar{p}(\overline{\operatorname{div} \delta \mathbf{a}}-\underline{\operatorname{div}} \delta \mathbf{a})+r \mathbf{A}: \delta \mathbf{A} \mathrm{d} V=0
\end{gathered}
$$

Equations (52) will serve as the basis of the discretized form of equilibrium. These equations differ from those presented by Armero [28].

\subsection{Approximated dilatation field}

The full quadrature of $J$, in Equation (39), has a penalizing effect in the analytical calculations carried out to linearize that term, as it will become apparent. Additionally, the numerical evaluation of that integral is generally approximated, resulting an approximated value for $\theta$. This is particularly relevant as $J=\operatorname{det}[\mathbf{F}]$ is a rational function of the element's co-ordinates.

An alternative approximation, distinct from the reduced selective integration previously discussed for the standard four-node element, is the reduced integration of the Jacobian $J$, whose value in the element's centre is denoted as $J_{0}$.

Using this approximated integration (which is not necessarily less accurate than the full integration), the dilatation can be determined as

$$
\theta=\operatorname{det}\left[\nabla_{0} \mathbf{x}+\mathbf{A}\right]_{0}=J_{0}
$$

Besides these motivations, there exists an intrinsic difficulty related to the enhancement of the deformation gradient by the term A. Generally, it appears to be difficult to obtain the displacement field related to $\mathbf{F}=\nabla_{0} \mathbf{x}+\mathbf{A}$, since $\mathbf{A}$ is generally independent of any displacement field. In this paper, only the element Qi5B-bar allows such a relation, because the additional enhanced mode is the bubble mode.

It is therefore useful, according to the described approximation, to define a particular form of $\mathbf{A}$ that has the property of being null in the element's centre. This condition is fulfilled by the two proposed elements. 


\section{DISCRETIZATION}

The discretized form for a co-ordinate $i$ of $\mathbf{x}$, denoted as $x_{i}$ is written as $x_{i}=N^{K} x_{i}^{k}, N^{k}$ being the shape function of node $k: N^{k}=\frac{1}{4}\left(1+\xi_{1}^{k} \xi_{1}\right)\left(1+\xi_{2}^{k} \xi_{2}\right)$, with $\xi_{1}$ and $\xi_{2}$ being the local co-ordinates and $x_{i}^{k}$ a nodal variable.

The spatial derivatives of $\delta x_{i}$ can be calculated as

$$
\delta x_{i, j}=N_{j}^{k} \delta x_{i}^{k}
$$

and the shape function derivative is written as

$$
N_{j}^{k}=\frac{\partial N^{k}}{\partial x_{j}}=\frac{\partial N^{k}}{\partial \xi_{l}} \frac{\partial \xi_{l}}{\partial x_{j}}
$$

where $\partial \xi_{l} / \partial x_{j}$ is calculated using the chain rule

$$
\frac{\partial x_{j}}{\partial \xi_{l}}=\frac{\partial N^{m}}{\partial \xi_{l}} x_{j}^{m}
$$

The enhanced term $\mathbf{A}$ can be determined through the internal variables denoted as $\alpha_{i}^{k}$ :

$$
A_{i j}=\alpha_{i}^{k} G_{j}^{k}
$$

Equation (57) defines the term $\mathbf{A}$ as a linear combination of the internal variables. The terms identified as $G_{j}^{k}$ in (57) are given interpolation functions.

In the work of Armero [28], the form adopted for $\mathbf{A}$ is far more involved, as it uses a central evaluation of the deformation gradient, and was first proposed by Glaser and Armero [12].

The related spatial tensor, a, can be written as

$$
a_{i j}=A_{i k} F_{k j}^{-1}=\alpha_{i}^{l} g_{j}^{l}
$$

A matrix $\mathbf{G}_{n d i m \times n e n h}$, whose dimension $n$ dim is the spatial dimension of the problem and dimension nenh is the number of additional deformation modes, can be defined with the scalar components $G_{j}^{k}$.

The construction of $\mathbf{A}$ is carried out in the isoparametric domain following the steps described by Simo et al. [3] but using an exact tensorial transformation. The final form is simply:

$$
\mathbf{G}=\mathbf{J}^{-\mathrm{T}} \mathbf{E}
$$

where $\mathbf{J}$ is the Jacobian matrix of the transformation between the local co-ordinates and the material co-ordinates: $\xi_{i} \rightarrow X_{j}$. Its scalar components can be written as $J_{i j}=\partial X_{i} / \partial \xi_{j}$.

It is important to note that, as shown by César de Sá and Natal Jorge [4], the proposed interpolation functions satisfy the following equation:

$$
\int_{\mathbb{B}} \mathbf{G} \mathrm{d} V=\mathbf{0}
$$

so that the elements possess a zero mean $\mathbf{A}$ over the domain, as imposed by Simo and Armero [7]. 
It is noticeable that this condition is satisfied without the need for evaluating the Jacobian in the elements centre which is the case for the standard enhanced strain elements [7,3].

The interpolation matrix $\mathbf{E}$ proposed in this paper has one of the two possible forms:

$$
\mathbf{E}_{Q i 5}=\left[\begin{array}{l}
-2 \xi_{1}\left(1-\xi_{2}^{2}\right) \\
-2 \xi_{2}\left(1-\xi_{1}^{2}\right)
\end{array}\right]
$$

or

$$
\mathbf{E}_{Q i 6}=\left[\begin{array}{cc}
-2 \xi_{1}\left(1-\xi_{2}^{2}\right) & 0 \\
0 & -2 \xi_{2}\left(1-\xi_{1}^{2}\right)
\end{array}\right]
$$

written for the Qi5B-bar and Qi6B-bar elements, respectively. These denominations are an extension of those introduced in Reference [4]. As already mentioned, the mode related to the form (61a) can be identified as a bubble mode. In the work of Armero [28], a two variable interpolation matrix is used.

Both the interpolation matrices (61) have the additional property of being null at the elements centre. Hence, the following statement is valid:

$$
\left.\mathbf{G}\right|_{\xi_{1}=0, \xi_{2}=0}=\mathbf{0}
$$

This property allows us to write Equation (53) as

$$
\theta=\left.\operatorname{det}\left[\nabla_{0} \mathbf{x}\right]\right|_{\xi_{1}=0, \xi_{2}=0}
$$

The divergence operators and the average divergence operators can be written in the following discretized form:

$$
\begin{aligned}
& \underline{\underline{\operatorname{div}} \delta \mathbf{a}}=\delta \alpha_{i}^{l} g_{i}^{l} \\
& \overline{\operatorname{div} \delta \mathbf{a}}=\delta \alpha_{i}^{l} g_{0 i}^{l}=0 \\
& \operatorname{div} \delta \mathbf{x}=\delta x_{i}^{l} N_{i}^{l} \\
& \overline{\operatorname{div} \delta \mathbf{x}}=\delta x_{i}^{l} N_{0 i}^{l}
\end{aligned}
$$

where, according to relation (63),

$$
\begin{aligned}
& \left.N_{0 k}^{l} \equiv N_{k}^{l}\right|_{\xi_{1}=0, \xi_{2}=0} \\
& \left.g_{0 k}^{l} \equiv g_{k}^{l}\right|_{\xi_{1}=0, \xi_{2}=0}=0
\end{aligned}
$$

In agreement with these particular forms, the discretized equations derived from the equilibrium equations (52) can be written as

$$
\begin{array}{r}
\int_{\mathbb{B}} \tau_{i j}\left[N_{j}^{k} \delta x_{i}^{k}+\frac{\delta_{i j}}{3}\left(N_{0 m}^{l} \delta x_{m}^{l}-N_{m}^{l} \delta x_{m}^{l}\right)\right] \mathrm{d} V+\delta \Pi_{\mathrm{ext}}=0 \\
\int_{\mathbb{B}} \tau_{i j}\left(g_{j}^{k} \delta \alpha_{i}^{k}-\frac{\delta_{i j}}{3} g_{m}^{l} \delta \alpha_{m}^{l}\right)+r G_{r}^{n} G_{r}^{p} \alpha_{s}^{n} \delta \alpha_{s}^{p} \mathrm{~d} V=0
\end{array}
$$

An inspection of the discretized forms (69) allows the conclusion that the discrete strain operator is the so-called B-bar matrix discussed in References $[17,16]$. Therefore, the proposed 
elements are of the B-bar type. The internal force vector for node $k$ can be straightforwardly obtained as a consequence of the discretized equations (69).

\section{LINEARIZATION}

To ensure the asymptotically quadratic convergence rate in the Newton-Raphson method, an exact linearization of the equilibrium equations (52) is carried out.

A rate analogy is used, so that the rates can be related to the iterative variations in the $\mathbf{x}$ and $\alpha$ variables:

$$
\begin{aligned}
& \mathbf{v} \Delta t=\Delta \mathbf{x} \\
& \dot{\alpha} \Delta t=\Delta \boldsymbol{\alpha}
\end{aligned}
$$

where $\mathbf{v}$ is the spatial velocity nodal vector and $\dot{\alpha}$ is the internal variable time derivative.

Equations (52) are linearized according to

$$
\delta \dot{\Pi} \Delta t \cong-\delta \Pi
$$

The final result is obtained using the chain rule. The discretized rates can be determined by some straightforward calculations, as follows.

The time derivative of the Kirchhoff stress tensor is calculated using the Truesdell rate, which is denoted as $\widetilde{\tau_{\mathrm{T}}}$.

The Truesdell rate of the Kirchhoff stress can be related to the time derivative of Kirchhoff stress as in Reference [36]:

$$
\dot{\tilde{\tau}}=\tilde{\tau_{\mathrm{T}}}+\mathbf{l} \tilde{\tau}+\tilde{\tau} \mathbf{l}^{\mathrm{T}}
$$

where $\mathbf{I}$ is the spatial velocity gradient whose co-ordinates are $l_{i j}=\partial v_{i} / \partial x_{j}$.

The Truesdell rate can be related to the deformation rate tensor through the spatial elasticity tensor, c, according to the following product:

$$
\widetilde{\tau_{\mathrm{T}}}=\mathbf{c}: \dot{\varepsilon}
$$

where $\dot{\boldsymbol{\varepsilon}}=\operatorname{sym}(\mathbf{l})$ is the deformation rate.

The spatial velocity gradient is calculated by its definition (noting that $\mathbf{l}$ is consistent with $\tilde{\tau}$, i.e. assumed) as follows:

$$
\mathbf{l}=\dot{\tilde{\mathbf{F}}} \tilde{\mathbf{F}}^{-1}=\left(\frac{\dot{\theta}}{3 \theta}-\frac{j}{3 J}\right) \mathbf{I}+\dot{\mathbf{f}}=\frac{1}{3}(\overline{\operatorname{div} \mathbf{v}}-\operatorname{div} \mathbf{v}+\overline{\operatorname{div} \dot{\mathbf{a}}}-\underline{\operatorname{div}} \dot{\mathbf{a}}) \mathbf{I}+\dot{\mathbf{f}}
$$

where $\dot{\mathbf{f}}=\dot{\mathbf{F}} \mathbf{F}^{-1}$ is an auxiliary term and $\dot{\mathbf{F}}=\nabla_{0} \mathbf{v}+\dot{\mathbf{A}}$ is the time derivative of the enhanced deformation gradient.

The deformation rate is simply

$$
\dot{\boldsymbol{\varepsilon}}=\frac{1}{3}(\overline{\operatorname{div} \mathbf{v}}-\operatorname{div} \mathbf{v}+\overline{\operatorname{div} \dot{\mathbf{a}}}-\underline{\operatorname{div}} \dot{\mathbf{a}}) \mathbf{I}+\frac{1}{2}\left(\dot{\mathbf{f}}+\dot{\mathbf{f}}^{\mathrm{T}}\right)
$$


The time derivative of $\nabla \delta \mathbf{x}$ can be calculated using the chain rule:

$$
\nabla \dot{\delta} \mathbf{x}=\nabla_{0} \delta \mathbf{x} \dot{\mathbf{F}}^{-1}=-\nabla \delta \mathbf{x} \dot{\mathbf{f}}
$$

and for the time derivative of $\delta \mathbf{a}$, the result is similar:

$$
\dot{\delta} \mathbf{a}=-\delta \mathbf{a} \dot{\mathbf{f}}
$$

The assumed pressure term $\bar{p}$ can be related to $\tilde{\tau}$ using Equation (50):

$$
\bar{p}=\frac{1}{3} \tilde{\mathbf{P}}: \tilde{\mathbf{F}}=\frac{1}{3} P_{i j} F_{i j}=\frac{1}{3} \tau_{i k} F_{j k}^{-1} F_{i j}=\frac{1}{3} \tau_{i k} \delta_{i k}=\frac{1}{3} \tilde{\tau}: \mathbf{I}
$$

so that its time derivative is simply:

$$
\dot{\bar{p}}=\frac{1}{3} \dot{\tilde{\tau}}: \mathbf{I}
$$

The time derivatives of the divergence operators are calculated performing the contraction between the identity tensor and the related gradients.

After some calculations it is possible to write the following relations:

$$
\begin{aligned}
& \underline{\operatorname{div}} \delta \mathbf{a}=-\delta \mathbf{a}^{\mathrm{T}}: \dot{\mathbf{f}} \\
& \operatorname{div} \delta \mathbf{x}=-\nabla \delta \mathbf{x}^{\mathrm{T}}: \dot{\mathbf{f}}
\end{aligned}
$$

The time derivatives of the average divergence operators $\overline{\operatorname{div} \delta \mathbf{x}}$ and $\overline{\operatorname{div} \delta \mathbf{a}}$, are determined using the previous relations (80) and the chain rule.

For $\overline{\operatorname{div} \delta \mathbf{x}}$, the following equation can be written as

$$
\overline{\operatorname{div} \delta \mathbf{x}}=-\frac{\dot{\theta}}{\theta^{2} V} \int_{\mathbb{B}} J \operatorname{div} \delta \mathbf{x} \mathrm{d} V+\frac{1}{\theta V} \int_{\mathbb{B}} \dot{J} \operatorname{div} \delta \mathbf{x} \mathrm{d} V+\frac{1}{\theta V} \int_{\mathbb{B}} J \operatorname{div} \delta \mathbf{x} \mathrm{d} V
$$

Using the derivative of $\operatorname{div} \delta \mathbf{x}$ in Equation (80b) and noting the following relations:

$$
\dot{\theta}=\theta(\overline{\operatorname{div} \mathbf{v}}+\overline{\operatorname{div} \dot{\mathbf{a}}})
$$

and

$$
\dot{J}=J(\operatorname{div} \mathbf{v}+\underline{\operatorname{div}} \dot{\mathbf{a}})
$$

it is possible to write the final result for $\overline{\operatorname{div} \delta \mathbf{x}}$ :

$\overline{\operatorname{div} \delta \mathbf{x}}=-(\overline{\operatorname{div} \mathbf{v}}+\overline{\operatorname{div} \dot{\mathbf{a}}}) \overline{\operatorname{div} \delta \mathbf{x}}+\frac{1}{\theta V} \int_{\mathbb{B}}(J \operatorname{div} \mathbf{v}+J \underline{\operatorname{div}} \dot{\mathbf{a}}) \operatorname{div} \delta \mathbf{x} \mathrm{d} V-\frac{1}{\theta V} \int_{\mathbb{B}} J \nabla \delta \mathbf{x}^{\mathrm{T}}: \dot{\mathbf{f}} \mathrm{d} V$

Equation (83) is visibly distinct from the equation derived by Simo et al. [17].

The difference is not only the absence of the field $\mathbf{a}$ in that paper [17], but even the linearized average spatial divergence is clearly different.

Introducing the approximation discussed in Section 4.5 , the result is much simpler:

$$
\overline{\operatorname{div} \delta \mathbf{x}}=-\nabla \delta \mathbf{x}_{0}^{\mathrm{T}}: \dot{\mathbf{f}}_{0}
$$




$$
\overline{\operatorname{div} \delta \mathbf{a}}=-\delta \mathbf{a}_{0}^{\mathrm{T}}: \dot{\mathbf{f}}_{0}
$$

where the subscript 0 indicates that the quantity is evaluated at the element's central point.

Using these results, the time derivative of the Kirchhoff stress tensor can be written as

$$
\dot{\tilde{\tau}}=\mathbf{c}:\left[\left(\frac{\dot{\theta}}{3 \theta}-\frac{\dot{J}}{3 J}\right) \mathbf{I}+\dot{\mathbf{f}}\right]+\frac{2}{3}\left(\frac{\dot{\theta}}{\theta}-\frac{\dot{J}}{J}\right) \tilde{\tau}+\dot{\mathbf{f}} \tilde{\tau}+\tilde{\tau} \dot{\mathbf{f}}^{\mathrm{T}}
$$

It is now appropriate to introduce the discretized results, necessary for the numerical implementation.

The auxiliary term $\dot{\mathbf{f}}$ can be written as

$$
\dot{\mathbf{f}}=\nabla \mathbf{v}+\dot{\mathbf{a}} \Leftrightarrow \dot{f}_{i j}=N_{j}^{k} v_{i}^{k}+g_{j}^{l} \dot{\alpha}_{i}^{l}
$$

And the term $\delta \dot{a}_{i j}$ can be discretized as

$$
\delta \dot{a}_{i j}=-\delta a_{i k} \dot{f}_{k j}=-\delta a_{i k}\left(N_{j}^{m} v_{k}^{m}+g_{j}^{l} \dot{\alpha}_{k}^{l}\right)
$$

The time derivatives of the discretized forms of the spatial divergence operators and divergence averages are written as follows:

$$
\begin{aligned}
& \underline{\operatorname{div} \delta \mathbf{a}}=-\delta \alpha_{j}^{l} g_{i}^{l}\left(N_{j}^{k} v_{i}^{k}-g_{j}^{m} \dot{\alpha}_{i}^{m}\right) \\
& \frac{\cdot}{\operatorname{div} \delta \mathbf{a}}=0 \\
& \operatorname{div} \delta \mathbf{x}=-\delta x_{j}^{l} N_{i}^{l}\left(N_{j}^{k} v_{i}^{k}-g_{j}^{m} \dot{\alpha}_{i}^{m}\right) \\
& \frac{\cdot}{\operatorname{div} \delta \mathbf{x}}=-\delta x_{j}^{l} N_{0 i}^{l} N_{0 j}^{k} v_{i}^{k}
\end{aligned}
$$

Therefore, the final discretized form of the time derivative of the Kirchhoff stress tensor can be written as

$$
\begin{aligned}
\dot{\tilde{\tau}}_{i j}= & c_{i j k l}\left\{\frac{1}{3} \delta_{k l}\left[-g_{p}^{l} \dot{\alpha}_{p}^{l}+\left(N_{0 q}^{m}-N_{q}^{m}\right) v_{q}^{m}\right]+\frac{1}{2}\left(N_{k}^{m} v_{l}^{m}+N_{l}^{n} v_{k}^{n}+g_{l}^{s} \dot{\alpha}_{k}^{s}+g_{k}^{r} \dot{\alpha}_{l}^{r}\right)\right\} \\
& +\frac{2}{3}\left[-g_{p}^{l} \dot{\alpha}_{p}^{l}+\left(N_{0 q}^{m}-N_{q}^{m}\right) v_{q}^{m}\right] \tau_{i j}+\left(N_{k}^{m} v_{i}^{m}+g_{k}^{l} \dot{\alpha}_{i}^{l}\right) \tau_{k j}+\left(N_{k}^{m} v_{j}^{m}+g_{k}^{l} \dot{\alpha}_{j}^{l}\right) \tau_{i k}
\end{aligned}
$$

and the linearized form for the assumed pressure is written as

$$
\dot{\bar{p}}=\dot{\tilde{\tau}}_{i i}
$$

Finally, the term $\dot{\mathbf{A}}$ is calculated as

$$
\dot{A}_{i j}=\dot{\alpha}_{i}^{k} G_{j}^{k}
$$

from which results a particularly simple form for the stabilizing term in the stiffness matrix. 
Now the following auxiliary notations are introduced:

$$
\widehat{\nabla \delta \mathbf{x}}=\nabla \delta \mathbf{x}+\frac{1}{3}(\overline{\operatorname{div} \delta \mathbf{x}}-\operatorname{div} \delta \mathbf{x})
$$

and

$$
\widehat{\delta \mathbf{a}}=\delta \mathbf{a}+\frac{1}{3}(\overline{\operatorname{div} \delta \mathbf{a}}-\underline{\operatorname{div}} \delta \mathbf{a})
$$

From which the linearized form of Equations (52) can be written as follows:

$$
\begin{gathered}
\int_{\mathbb{B}} \dot{\boldsymbol{\varepsilon}}: \mathbf{c}: \widehat{\nabla \delta \mathbf{x}}+\left(\mathbf{l} \tilde{\tau}+\tilde{\tau} \mathbf{l}^{\mathrm{T}}\right): \widehat{\nabla \delta \mathbf{x}} \\
\quad+\tilde{\tau}:\left[-\nabla \delta \mathbf{x} \dot{\mathbf{f}}+\frac{1}{3} \mathbf{I}\left(-\nabla \delta \mathbf{x}_{0}^{\mathrm{T}}: \dot{\mathbf{f}}_{0}+\nabla \delta \mathbf{x}^{\mathrm{T}}: \dot{\mathbf{f}}\right)\right] \mathrm{d} V+\delta \dot{\Pi}_{\mathrm{ext}}=-\delta \Pi(\delta \mathbf{x}) / \Delta t \\
\int_{\mathbb{B}} \dot{\boldsymbol{\varepsilon}}: \mathbf{c}: \widehat{\delta \mathbf{a}}+\left(\mathbf{l} \tilde{\tau}+\tilde{\tau} \mathbf{l}^{\mathrm{T}}\right): \widehat{\delta \mathbf{a}}+\tilde{\tau}:\left[-\delta \mathbf{a} \dot{\mathbf{f}}+\frac{1}{3} \mathbf{I}\left(\delta \mathbf{a}^{\mathrm{T}}: \dot{\mathbf{f}}\right)\right]+r \dot{\mathbf{A}}: \delta \mathbf{A} \mathrm{d} V=-\delta \Pi(\delta \mathbf{a}) / \Delta t
\end{gathered}
$$

The stiffness matrix is straightforwardly derived from Equations (91) and the discussed time derivatives.

\section{STABILIZATION PARAMETER}

The proposed stabilization term, dependent on the $r$ parameter, allows the control of the instability modes (i.e. hourglass modes) often present in the element formulations of the enhanced strain type.

For some element formulations, based on reduced integration or uniform integration (as, for instance, the technique developed by Bonet and Bhargava [20]), there are some alternative ways of stabilizing the element which are also based on some sort of stabilizing term.

The experiences carried out by the authors of this paper, with the present elements, allow the conclusion that the technique gives an efficient control of the element's instabilities. For the element that possesses 4 internal variables, denoted as Qi6B-bar, the following constant stabilization parameter, $r$ is proposed:

$$
r=\frac{1}{100} \mu
$$

$\mu$ being the shear modulus of the material.

Usually, this value does not increase too much the element's hourglass stiffness to the point of damaging the intrinsic good results, but allows the control of instabilities otherwise present in several tests.

The technique suggested by Bonet and Bhargava [20], was also tested with successful results, particularly for regular meshes.

However, the proposed technique has a natural justification for enhanced strain elements and has a variational support.

It seems appropriate to refer that the Qi6B-bar element, in the absence of a stabilizing term possesses a marked stability deficiency. However, the element with two internal variables, 
denoted as Qi5B-bar, was tested here with a null $r$, with good results. A null $r$ is equivalent to the orthogonality condition set by Simo and Armero [7] between the tensorial Lagrange multiplier and the constraint $\mathbf{A}=\mathbf{0}$. This statement can be verified through Equation (22).

It is clear that the imposition of a constraint by a multiplier that is assumed to be orthogonal to the constraint is equivalent to not imposing the constraint.

\section{NUMERICAL EXAMPLES}

The following set of numerical examples attempts to validate the new proposed formulations and compare its results with those obtained using well established techniques. A variety of situations is tested, which allow to get a grasp of the elements' behaviour, namely in compression, tension and bending situations.

The presented examples are two-dimensional and obey to the plane strain hypothesis, in agreement with the discussion of Section 4.1.

The Newton-Raphson method is employed, with automatic load increasing and line-search.

Beginning with the second load-step, the predictor displacement is calculated as a quadratic extrapolation of the displacements, and possesses the peculiarity of including a stiffness matrix decomposition:

$$
{ }^{n+1} \Delta \mathbf{u}={ }^{n-1} \mathbf{u} k_{1}+{ }^{n} \mathbf{u} k_{2}+{ }^{n} \mathbf{K}^{-1}\left({ }^{n+1} \mathbf{f}-{ }^{n} \mathbf{i}\right) k_{3}
$$

where ${ }^{n+1} \Delta \mathbf{u}$ is the displacement variation foreseen for the $n+1$ step. The terms ${ }^{n-1} \mathbf{u}$ and ${ }^{n} \mathbf{u}$ are the calculated displacements at the penultimate and last steps, respectively.

The ${ }^{n} \mathbf{K}$ matrix is the tangent stiffness matrix calculated at the end of the $n$th step, and the difference ${ }^{n+1} \mathbf{f}-{ }^{n} \mathbf{i}={ }^{n} \Delta \Lambda \mathbf{f}$ is the first residual corresponding to the step $n+1$.

The parameters $k_{1}, k_{2}$ and $k_{3}$ are evaluated by the load factors at the steps $n$ and $n-1:{ }^{n} \Lambda$, ${ }^{n-1} \Lambda$, and by the load factor variation ${ }^{n} \Delta \Lambda$. Hence,

$$
\begin{aligned}
& k_{1}=z_{1} x_{1}+z_{2} x_{4}+z_{3} x_{7} \\
& k_{2}=-z_{1}+z_{1} x_{2}+z_{2} x_{5}+z_{3} x_{8} \\
& k_{3}=z_{1} x_{3}+z_{2} x_{6}+z_{3} x_{9}
\end{aligned}
$$

where

$$
\begin{aligned}
& x_{1}=\frac{{ }^{n} \Lambda^{2}}{y}, \quad x_{2}=\frac{{ }^{n-1} \Lambda^{2}-2^{n-1} \Lambda^{n} \Lambda}{y} \\
& x_{3}=\frac{{ }^{n-1} \Lambda^{n} \Lambda^{2}-{ }^{n-1} \Lambda^{2}{ }^{n} \Lambda}{y}, \quad x_{4}=-2 \frac{{ }^{n} \Lambda}{y} \\
& x_{5}=2 \frac{{ }^{n} \Lambda}{y}, \quad x_{6}=\frac{{ }^{n-1} \Lambda^{2}-{ }^{n} \Lambda^{2}}{y} \\
& x_{7}=\frac{1}{y}, \quad x_{8}=-\frac{1}{y} \\
& x_{9}=\frac{{ }^{n} \Lambda-{ }^{n-1} \Lambda}{y} \quad y=\left({ }^{n} \Lambda-{ }^{n-1} \Lambda\right)^{2}
\end{aligned}
$$


and

$$
z_{1}=\frac{1}{{ }^{n} \Delta \Lambda} \quad z_{2}=z_{1}\left({ }^{n} \Lambda+{ }^{n} \Delta \Lambda\right) \quad z_{3}=z_{1}\left({ }^{n} \Lambda+{ }^{n} \Delta \Lambda\right)^{2}
$$

This extrapolation often allows a speed-up of the convergence rate in elasto-plastic problems, that can be irregular in the early iterations. However, if divergence occurs, this predictor is temporarily turned off.

\subsection{Material model}

The elasto-plastic $J_{2}$ model for finite strains is based on the multiplicative decomposition of the deformation gradient and on the assumption of existence of a hyperelastic potential. The assumed deformation gradient is, in this model, decomposed in agreement with the following hypothesis:

$$
\tilde{\mathbf{F}}=\tilde{\mathbf{F}}^{\mathrm{e}} \tilde{\mathbf{F}}^{\mathrm{p}}
$$

where $\tilde{\mathbf{F}}^{\mathrm{e}}$ is the elastic term and $\tilde{\mathbf{F}}^{\mathrm{p}}$ the plastic term of the assumed deformation gradient.

The proposed formulation corresponds to that developed by Simo in References [37, 32], in its spatial form. The yield condition is given by the equation $\varphi=\|\mathbf{s}\|-\sqrt{\frac{2}{3}}\left[Y_{0}+h\left(\varepsilon_{\mathrm{p}}\right)\right]=0$ where $\varepsilon_{\mathrm{p}}$ is the effective plastic strain and $\mathbf{s}$ is the deviatoric part of the Kirchhoff stress tensor.

The constant $Y_{0}$ is the initial yield stress.

The strain hardening function is the one presented in Reference [17]:

$$
h\left(\varepsilon_{\mathrm{p}}\right)=\left(Y_{\infty}-Y_{0}\right)\left[1-\mathrm{e}^{-\delta \varepsilon_{\mathrm{p}}}\right]+H \varepsilon_{\mathrm{p}}
$$

where $Y_{\infty}$ is the saturation stress, $\delta$ is the saturation exponent and $H$ is the linear hardening parameter.

The hyperelastic potential has an uncoupled form and can be written with the following notation, as referred in Reference [9] (distinct from the hyperelastic potential proposed by Simo et al. $[36,7])$ :

$$
W=\kappa U\left(J^{\mathrm{e}}\right)+\bar{W}\left(\overline{\mathbf{b}^{\mathrm{e}}}\right)
$$

and

$$
U\left(J^{\mathrm{e}}\right)=\frac{1}{2}\left[\ln \left(J^{\mathrm{e}}\right)\right]^{2} \bar{W}\left(\overline{\mathbf{b}^{\mathrm{e}}}\right)=\frac{1}{2} \mu\left(\operatorname{tr}\left[\overline{\mathbf{b}^{\mathrm{e}}}\right]-3\right)
$$

where $\mathbf{b}^{\mathrm{e}}=\tilde{\mathbf{F}}^{\mathrm{e}} \tilde{\mathbf{F}}^{\mathrm{eT}}, \overline{\mathbf{b}^{\mathrm{e}}}=J^{\mathrm{e}-2 / 3} \mathbf{b}^{\mathrm{e}}$ and $J^{\mathrm{e}}=\operatorname{det}\left[\tilde{\mathbf{F}}^{\mathrm{e}}\right]$.

The elastic material constants $\kappa$ and $\mu$ are the standard bulk modulus and shear modulus, respectively.

This material model is analytically simple and, due to the use of the radial return algorithm (for implementation details, consult Reference [36]) is computationally efficient.

\subsection{Nomenclature of the tested elements}

In the examples given here, there are 6 different element formulations.

The formulations are based on the calculations presented and on References [4, 7]. A $2 \times 2$ point, Gauss integration rule is used for all the formulations. Although this type of integration 


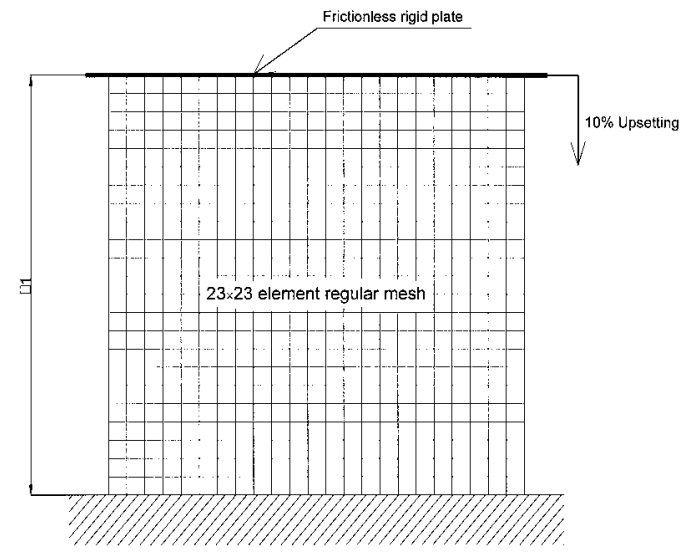

Figure 1. Block compression; geometry, finite element mesh and boundary conditions.

is often said to be reduced for enhanced strain formulations (as discussed by Simo et al. [3]), it is known that the instabilities do not disappear completely (as illustrated by Glaser and Armero [12]) when the number of integration points is increased.

Due to this fact, the use of a higher quadrature rule was avoided, as it penalizes the computing times in plasticity problems.

The standard formulation of the four-node element is identified by the symbol Q4. The B-bar formulation, in agreement with the exposed formulation but without additional modes, is identified by the symbol Q4B-bar. The fundamental difference between this formulation and the well known B-bar element is the discussed (in Section 4.5) approximation of the dilatation field.

The well established enhanced strain formulation, originally proposed for geometrically non linear problems by Simo and Armero [7], is identified by its original denomination Q1E4.

The non-linear extension of the recently proposed element by César de Sá and Natal Jorge [4] is denoted Qi6.

The B-bar element with two internal variables, corresponding to the bubble mode, is identified as Qi5B-bar. The B-bar extension of the Qi6 element is named Qi6B-bar.

Finally, we note that the truncated Taylor expansion of the element-shape functions, as discussed in Reference [14] was tested. Some slight differences were noted for coarse meshes. However, the results presented were obtained with the standard-shape functions.

\subsection{Block upsetting}

This problem consists of a compression of an elasto-plastic block. The corresponding model is presented in Figure 1.

It is noticeable that a similar problem was inspected in a paper by Crisfield et al. [38], for an upsetting displacement, corresponding to 30 per cent of the block's height. In that paper the presence of hourglass instabilities was detected, although a five-point integration rule was used. A $5 \times 10$ element mesh was used, taking advantage of the existence of one symmetry plane. 

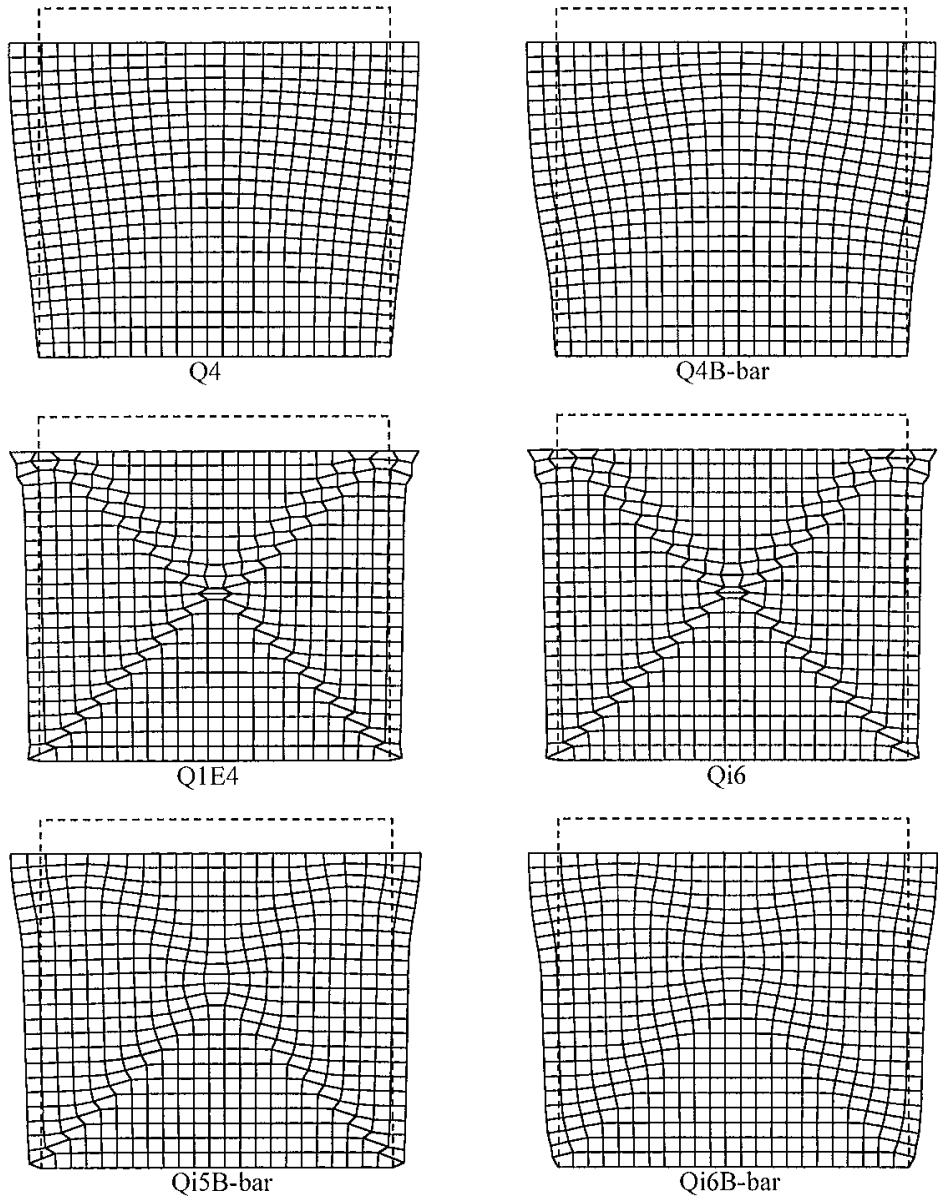

Figure 2. Block compression; deformed meshes of the 6 analysed element formulations.

Schonauer et al. [39], using an elasto-plastic formulation based on the Hencky strain tensor, managed to reduce the instabilities presented in Reference [38], maintaining the five-point quadrature rule.

The mesh proposed by Crisfield et al. appears to be (as discussed by Schonauer et al. [39]), excessively coarse to model adequately the shear band zone. With the mesh used in this work, it is possible to obtain those bands with just 10 per cent upsetting, as it will be verified.

The material properties are the same as in Crisfield et al., with shear modulus $\mu=92.53$ and bulk modulus $\kappa=200.47$. The yield stress is $Y_{0}=4.81$ and the material is considered to be elastic/perfectly plastic.

The deformed meshes related to the 6 formulations are exhibited in Figure 2. The presence of slight instabilities, on some mesh regions, exhibited by the Qi6 and Q1E4 elements is clear. The distortions present in these regions tend to increase with the imposed vertical displacement. It is also visible that all the other elements present less distorted meshes. The 


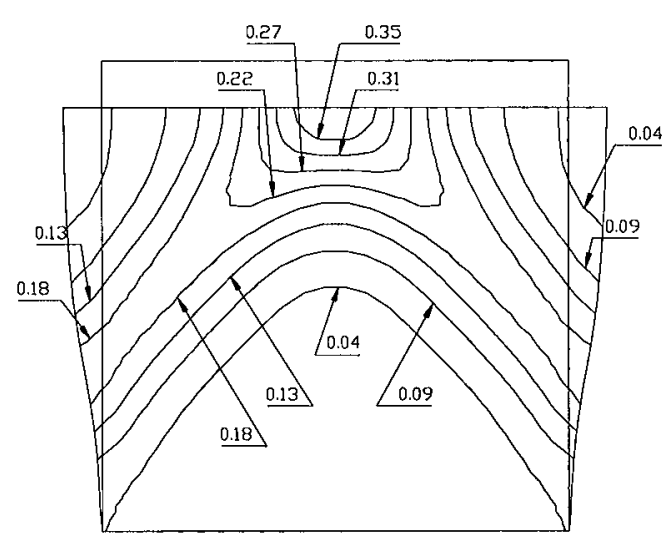

Figure 3. Block compression; contour plot of the effective plastic strain for the Q4B-bar element.

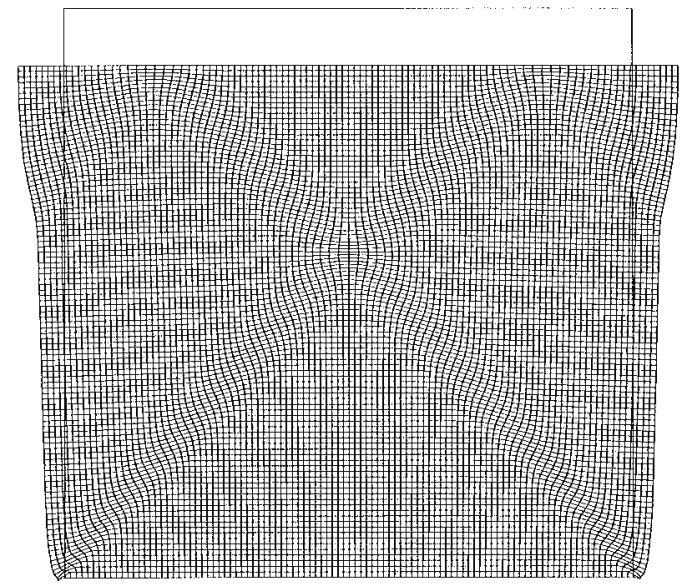

Figure 4. Block compression; $100 \times 100$ element deformed mesh for the Q4B-bar element.

Qi6B-bar element manifests a visible 'shear-band' deformation, in well defined areas. These areas are somewhat related to the concentration of the effective plastic strain, as it can be observed in the contour plot of Figure 3, which corresponds to the effective plastic strain obtained with the Q4B-bar element.

It is important to comment the apparent similarity between the results of the Q4 element and the other elements. In this type of tests, where the displacement is imposed, the volumetric locking effect is not explicitly apparent. An analysis of the reaction forces can be useful to conclude that these are far superior for the Q4 element. A reaction analysis was carried out by Wriggers and Hueck [14] for a similar problem (in the elastic range), comparing various formulations.

The instabilities exhibited by the Qi6 and Q1E4 formulations are not directly related to the effective plastic strain distribution, as the Q1E4 element presents the same symptoms in some purely elastic problems (as shown in References $[13,11]$ ).

Whether or not an hourglass free formulation can reproduce the shear bands is a relevant question. An analysis using a $100 \times 100$ element mesh and the Q4B-bar formulation allows the clear identification of the bands. Figure 4 shows the deformed mesh.

\subsection{Cook's membrane}

This test consists on a examination of the proposed elements performance, in situations of simultaneous shear and bending. The problem consists of a trapezoidal panel clamped on one side and subject to a shear load on the opposite side. Figure 5 displays the geometry and the boundary conditions of the problem analysed.

This problem is very common in both linear and non-linear analysis $[27,9,26,12]$.

In the present paper the material is considered elasto-plastic, with the following property values: bulk modulus $\kappa=164.21$; shear modulus $\mu=80.1983$; initial yield stress $Y_{0}=0.45$ and the hardening parameters $Y_{\infty}=0.715, \delta=16.93, H=0.12924$. 


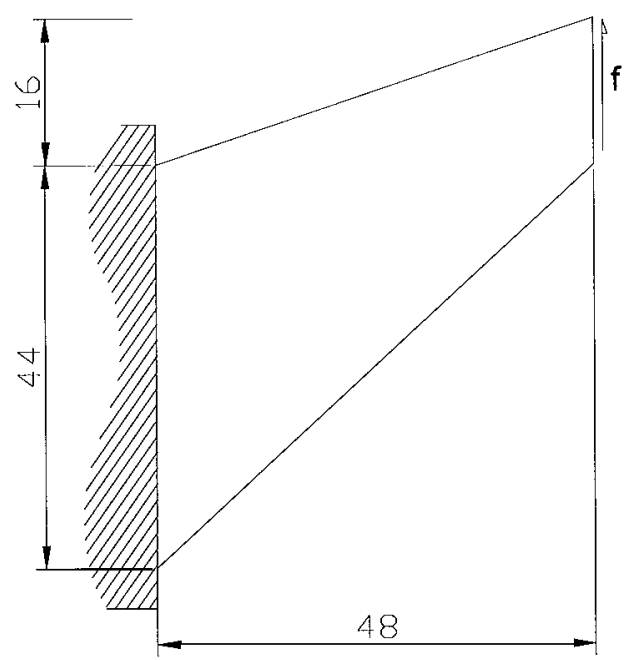

Figure 5. Cook's membrane; geometry and boundary conditions.

Table I. Top vertical displacement in the Cook's membrane problem for the tested elements.

\begin{tabular}{lcccccccc}
\hline & \multicolumn{7}{c}{ Number of elements in one side } \\
Formulation & 2 & 5 & 10 & 15 & 20 & 25 & 30 & 35 \\
\hline Q4 & 0.359 & 1.36 & 2.89 & 3.97 & 4.71 & 5.21 & 5.59 & 5.8 \\
Q1E4 & 3.80 & 6.35 & 6.82 & 6.88 & 6.93 & 6.95 & 6.97 & 6.97 \\
Qi6 & 4.63 & 6.58 & 6.83 & 6.89 & 6.95 & 6.96 & 6.97 & 6.98 \\
Q4B-bar & 2.71 & 5.85 & 6.51 & 6.74 & 6.81 & 6.86 & 6.91 & 6.92 \\
Qi5B-bar & 3.75 & 6.33 & 6.74 & 6.83 & 6.9 & 6.92 & 6.95 & 6.97 \\
Qi6B-bar & 6.05 & 6.77 & 6.71 & 6.91 & 6.92 & 6.94 & 6.96 & 6.97 \\
\hline
\end{tabular}

The identified total shear force $f$ has a value of 5 consistent units and is applied in variable force increments. This force is equally distributed by the extremity nodes and possesses a constant direction.

An analysis of the tip vertical displacement was carried out. The results obtained with that analysis are presented both in tabular (Table I) and chart form (Figure 6).

In the presented chart there is a plot of the results obtained with the Qi6, Q1E4, Qi6B-bar and Qi5B-bar elements, and the results for all the formulations are tabulated.

Observing Figure 6 and Table I presented, one can verify the very good results obtained with element Qi5B-bar (almost at the level obtained with the Q1E4 element) and with Qi6 element, whose performance is always superior or at least equal to the Q1E4. The Qi6B-bar element usually presents very good results, however, its convergence is non-monotonous due to the value obtained with the $10 \times 10$ mesh.

\subsection{Indentation problem}

This problem is inspired in the one carried out by Crisfield et al. [38] for a Mooney-Rivlin elastic material. 


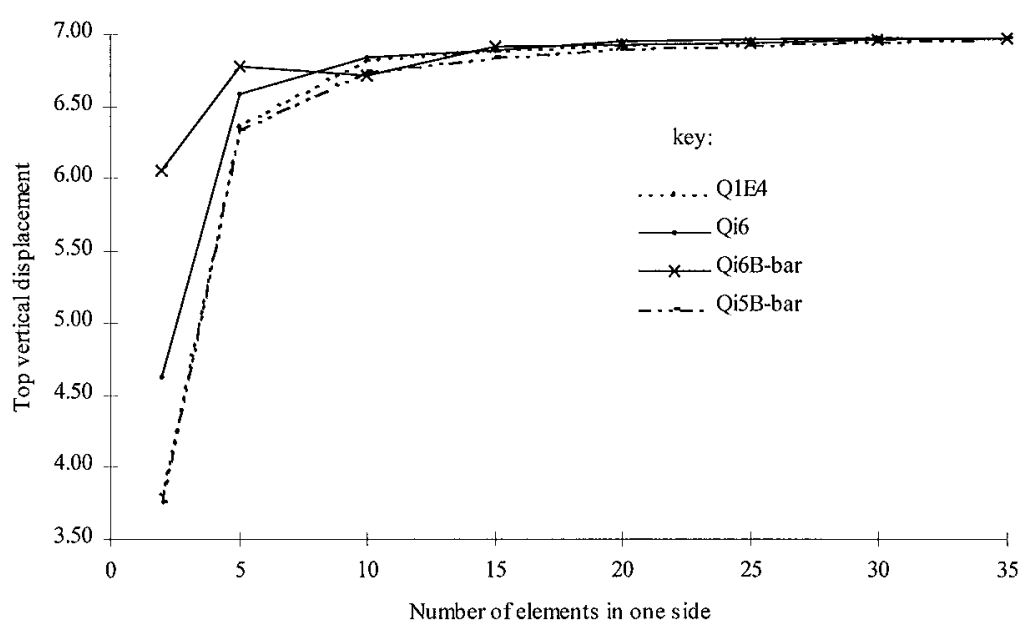

Figure 6. Cook's membrane; top vertical displacement convergence for several mesh densities.

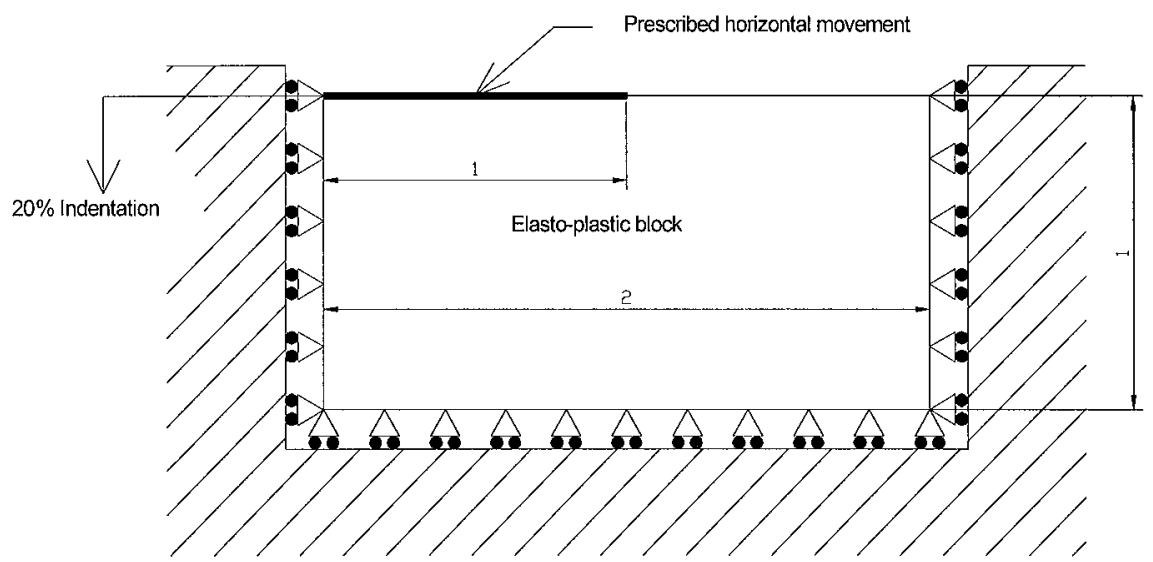

Figure 7. Block indentation; geometry and boundary conditions.

The test consists on a partially compressed block, with an indentation of 20 per cent of the total height (25 per cent in the elastic case described in the Reference [38]) by a friction plate. The block geometry and the boundary conditions are detailed in Figure 7.

The compression is applied by displacement control. Two materials are analysed: nearincompressible elastic and elasto-plastic, with the purpose of showing the qualitative differences in the instabilities.

The elastic material properties are bulk's modulus $\kappa=3.5 \times 10^{14}$ and shear modulus $\mu=7 \times$ $10^{9}$. This near-incompressible properties tend to force the distinction between elements.

For the elasto-plastic material, a lower bulk modulus is used; $\kappa=3.5 \times 10^{12}$ and the shear modulus is $\mu=7.047 \times 10^{9}$. This material is considered elastic/perfectly plastic and the yield stress is $Y_{0}=1 \times 10^{9}$. 

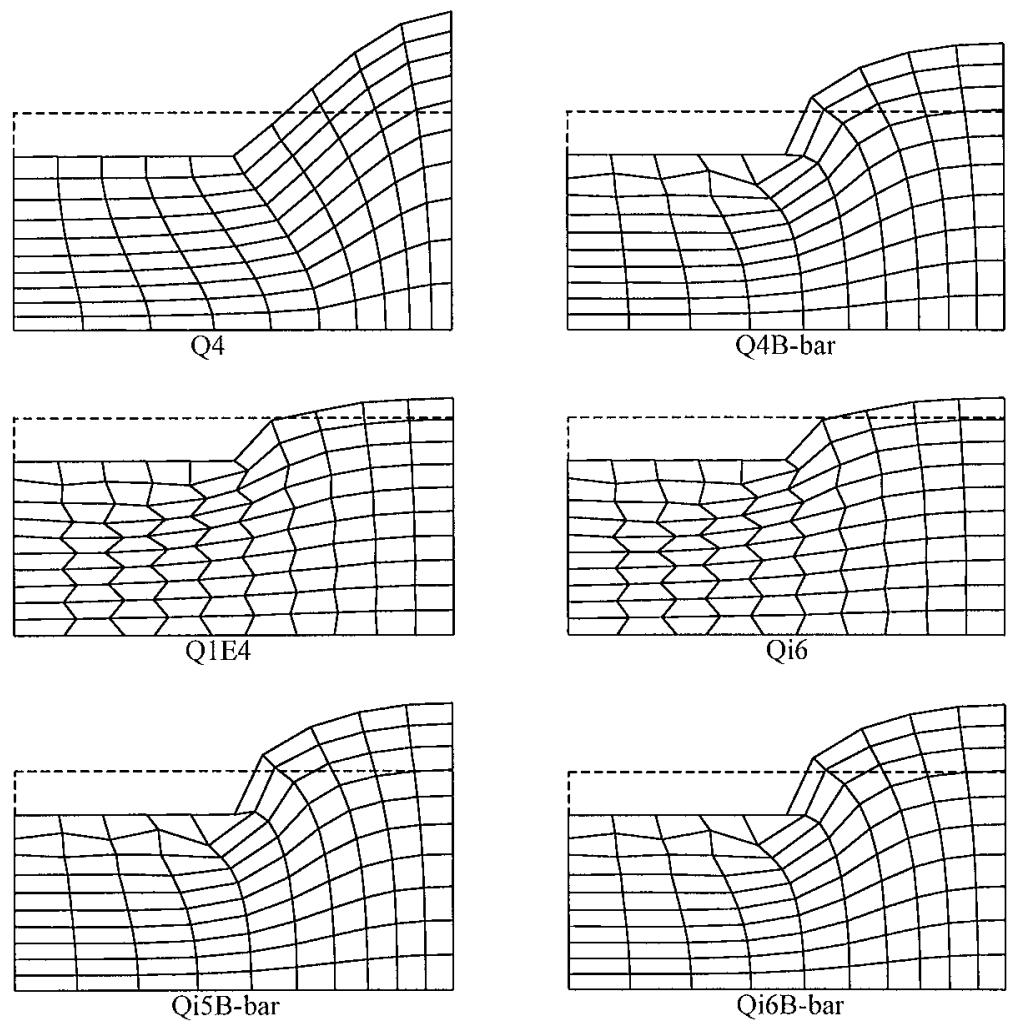

Figure 8. Block indentation; deformed meshes resulting from the elastic analysis.

The deformed meshes corresponding to the 6 analysed elements for the two problems, elastic and elasto-plastic, are presented in Figures 8 and 9, respectively.

For the elastic case, the hourglass patterns presented by the elements Qi6 and Q1E4 (and also the similarity between the obtained results) is noticeable. The violation of the nearincompressibility condition by these elements is clear, in finite strain situations, which presents a serious limitation to the general use of the standard enhanced strain elements.

The behaviour of the elements Q4B-bar, Qi5B-bar and Qi6B-bar is similar, with some distortion in critical zones. Nevertheless, these results are far more reasonable than those obtained with the Qi6 and Q1E4 elements.

In the elasto-plastic case the elements Q4B-bar, Qi5B-bar and Qi6B-bar present tiny differences between themselves, with larger distortions than those in the elastic case. The results obtained with elements Qi6 and Q1E4 are clearly unrealistic.

The apparently acceptable results exhibited by the Q4 element in the elasto-plastic case deserve a final comment. The vertical reactions in the indented zone are far larger than those presented by all the other elements, revealing a developing locking behaviour. A chart representing the vertical reactions along the indented zone for the 6 elements is presented in the Figure 10. 

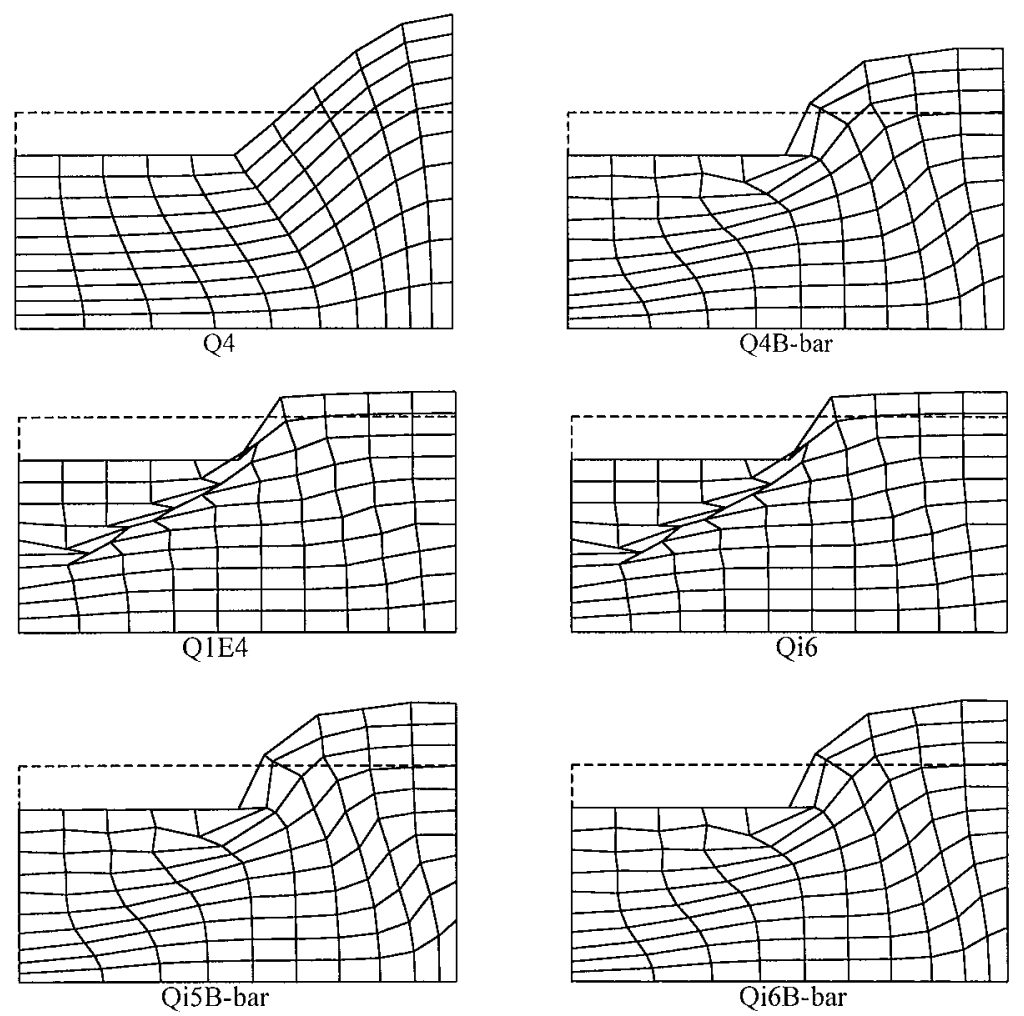

Figure 9. Block indentation; deformed meshes resulting from the elasto-plastic analysis.

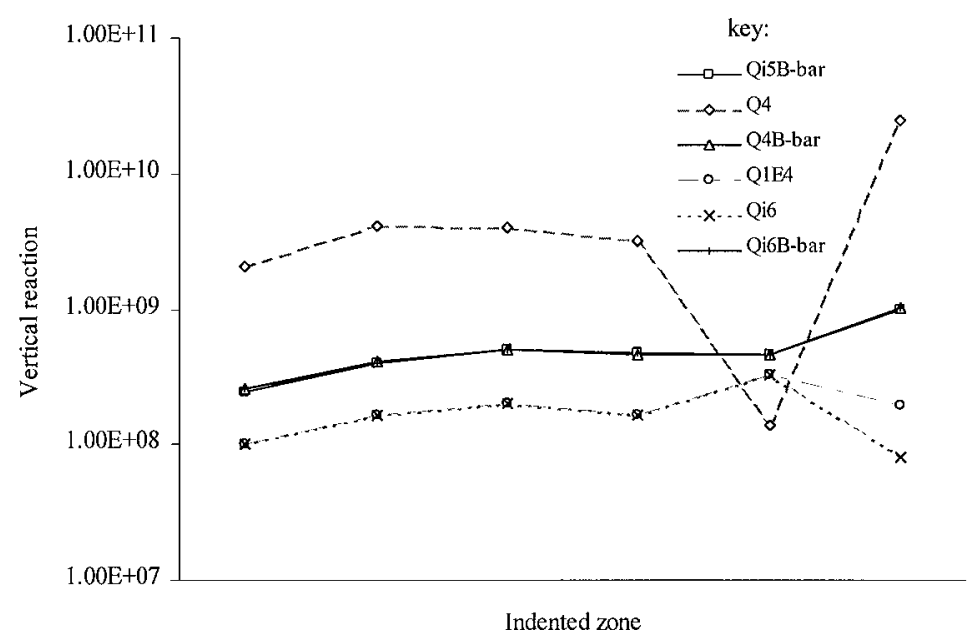

Figure 10. Block indentation; vertical reaction in the indented zone for the 6 analysed elements in the elasto-plastic analysis. 


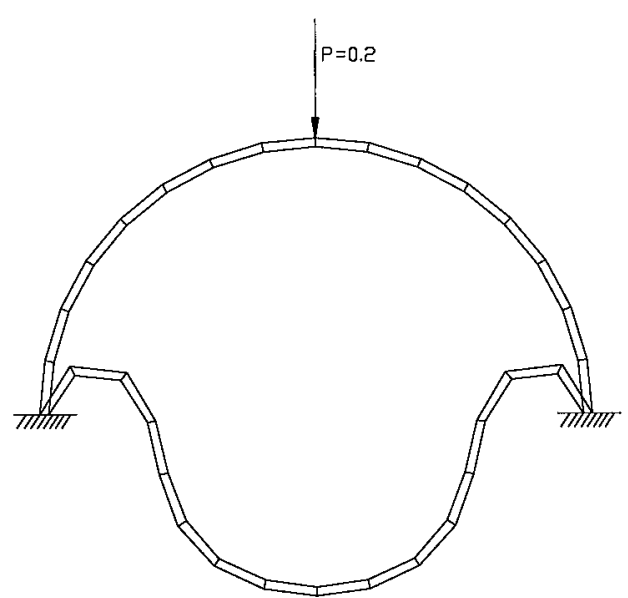

Figure 11. Elastic instability of a ring; original geometry, deformed geometry and boundary conditions.

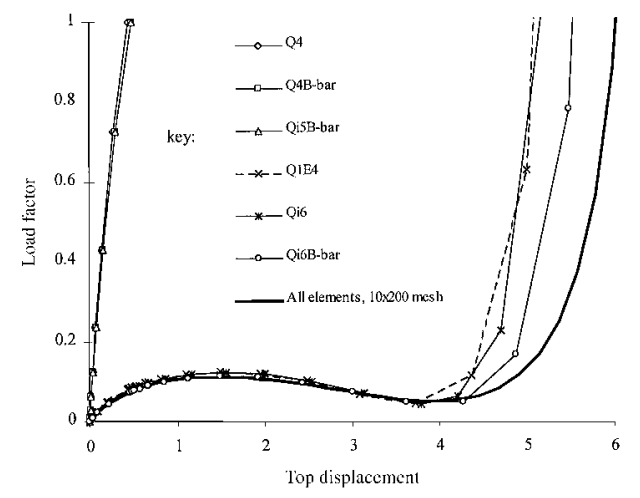

Figure 12. Elastic instability of a ring; load factor versus top displacement curve.

\subsection{Elastic stability of a circular ring}

In this example, the adequacy of these continuum elements to the analysis of a structural problem is examined.

With this purpose, a $180^{\circ}$ arch is analysed, with an interior radius of 3 and width 0.1 , subject to a vertical point force $P=0.2$ on its top. This arch is twice clamped in its extremities, as Figure 11 indicates.

This problem is particularly demanding for the tested elements, since they present a length/ width ratio that is admittedly penalizing (see, about the bending of a slender beam using this type of elements, Reference [21]).

The material is elastic, with the following properties: $\mu=80.77$ and $\kappa=175$. An alternative stabilizing parameter value of $r=6$ was adopted. The solution algorithm is the NewtonRaphson method with spherical arc-length control. The initial arc-length and its variation are automatic.

The 8 element mesh (due to the geometry's symmetry) was chosen to indicate the absence of critical load (in the interval $[0,0.2]$ ) when using the elements Q4, Q4B-bar and Qi5B-bar.

The charts with the load factor versus displacement curves corresponding to the point under the load are plotted for the 6 analysed elements in Figure 12. From the curves represented in Figure 12 it is possible to see the locking behaviour of the Q4, Q4B-bar and Qi5B-bar elements and the acceptable behaviour of the Qi6 and Q1E4 elements. The Qi6B-bar gives more accurate answers than the other elements in this test, when compared with the results obtained with a refined $10 \times 200$ mesh (half the arch).

For that mesh density, the load-displacement curves of the examined elements (including the Q4 element) are similar.

\subsection{Tension test}

This example consists on a tension test of a plane specimen, and has been widely published $[7,3,12,31]$. 


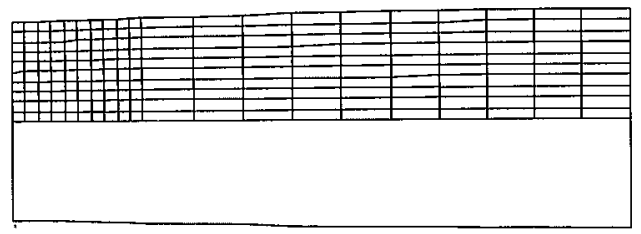

Q4
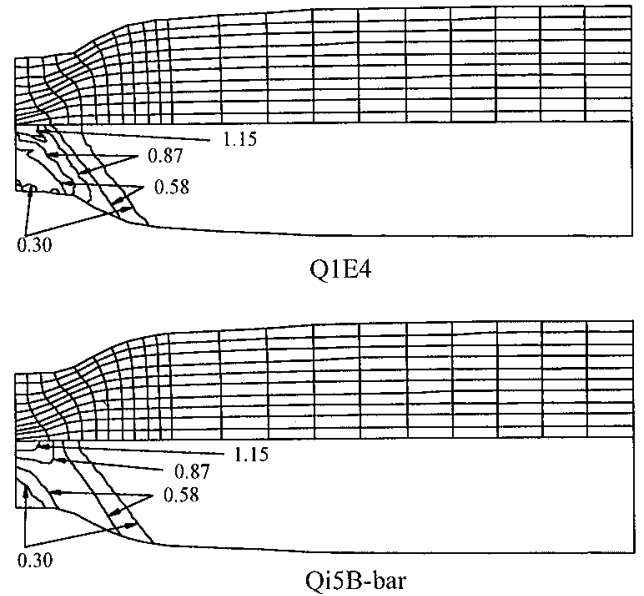

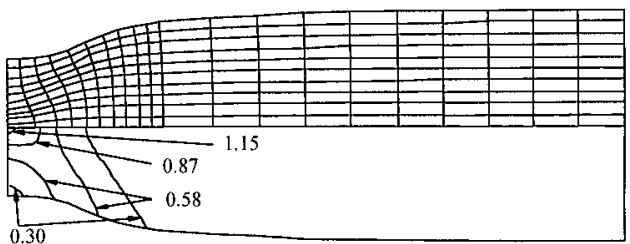

Q4B-bar
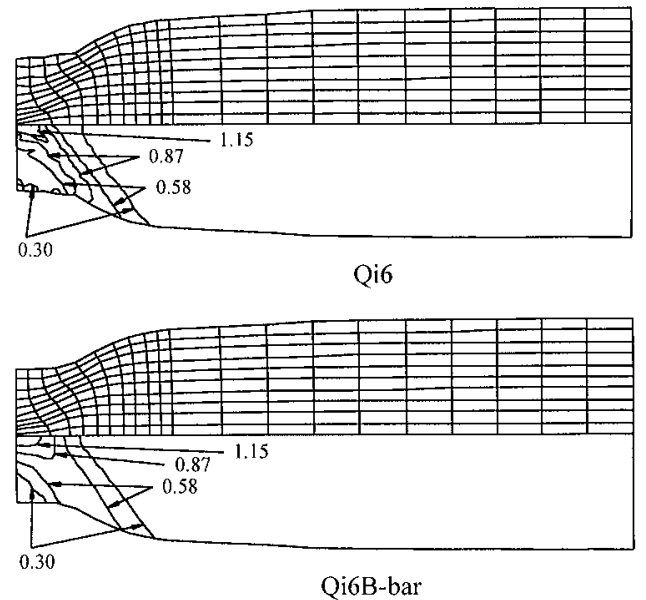

Figure 13. Plastic localization problem; deformed meshes and effective plastic strain contour plots.

The test allows the examination of the plastic necking zones. The necking zones present, with certain element formulations, several shear bands inclined $45^{\circ}$ in relation to the loading axis.

A 200 element mesh is used, with 12.826 width and 53.334 height, according to References $[7,31]$. An imperfection is introduced through the reduction of 1.8 per cent in the width at the specimen's centre (as described by de Souza Neto et al. [31]).

The specimen bar is discretized in $1 / 4$ of the geometry, owing to the presence of two symmetry planes. The analysis is carried out with displacement control with a total imposed stretch of $\bar{u}=4.7$.

The material properties are the same as in Reference [7], with the elastic properties $\kappa=164.21$, $\mu=80.1983$. The initial yield stress is $Y_{0}=0.45$ and the hardening properties are $Y_{\infty}=0.715$, $\delta=16.93, H=-0.012924$.

Using distinct material properties, Glaser and Armero [12] detected the existence of instabilities with the enhanced strain elements, even with five- and nine-point integration rule.

The deformed meshes related to the 6 examined elements are presented in Figure 13. The effective plastic strain contour plots are also represented. It is visible that the Qi6 and Q1E4 elements present some instabilities and the effective plastic strain contour plot shows some irregularity.

The Q4B-bar, Qi5B-bar and Qi6B-bar elements do not show any instabilities. It is also observable that the plastic areas in elements Qi5B-bar and Qi6B-bar present a higher effective plastic strain concentration than the one presented by Q4B-bar element. 


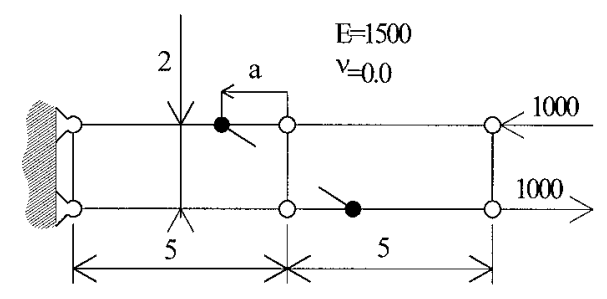

Figure 14. Geometry, boundary conditions and material properties for the mesh distortion test.

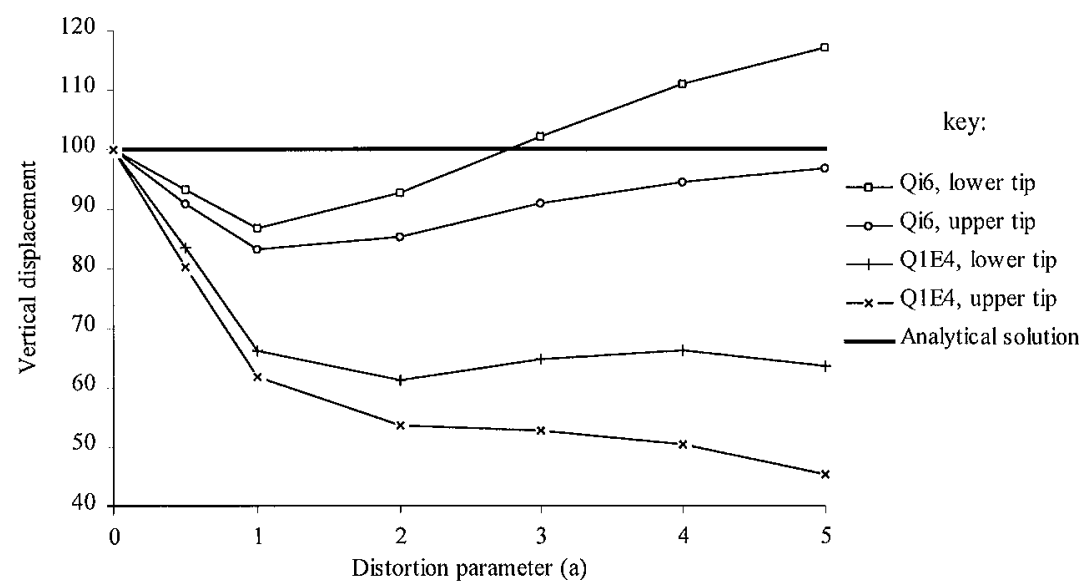

Figure 15. Vertical displacement of the nodes with applied forces, for various values of the distortion parameter.

In this case the Q4 element exhibits a severe locking behaviour.

\subsection{Mesh distortion test}

The purpose of this linear elastic test is the inspection of the bending behaviour of the Qi6 element, proposed by César de Sá and Natal Jorge [4], when compared with the standard enhanced strain element, introduced by Simo and Armero [7]. This comparison is particularly relevant as it has been recently stated by Lautersztajn and Samuelsson [8] that the Qi6 element performs rather poorly for plane bending. One other important aspect is the evaluation of the mesh distortion sensitivity of the two formulations in the bending case. Therefore, the bending test presented in Reference [8] is reproduced here.

Figure 14 shows the mesh, the boundary conditions and material properties of the test.

In Figure 15, the vertical displacement of the nodes with applied forces is plotted and compared with the result obtained using the beam theory.

It is clear that, in this test, the Qi6 element performs better than the original Q1E4 (incompatible) element. This conclusion somehow contradicts the statement referred in Reference [8]. 


\section{CONCLUSIONS}

In this paper two new elements have been described. These elements, named as Qi5B-bar and Qi6B-bar possess two and four internal variables, respectively. The enhanced deformation gradient, corresponding to an enhanced strain formulation is projected so that the dilatation is constant in the element's domain. The dilatation field results from an average reduced integration, with the purpose of imposing its independence from the internal variables. The variational basis of these elements is a four-field functional, which contains a stabilizing term that corresponds to a constraint nullifying the distortional part of the enhanced deformation gradient. Thus, it is more suited to the enhanced strain elements than other previously proposed stabilizers (as the one presented in Reference [20]). The enhanced deformation gradient is calculated with an exact tensorial transformation, as it does not need the usually adopted [7] procedure of evaluating the Jacobian matrix in the centre of the element.

With the use of a $J_{2}$ elasto-plastic material model, it is clear that both the Qi6 element (proposed by César de Sá and Natal Jorge [4]), and the Q1E4 element (proposed by Simo and Armero [7]) present pronounced instabilities in practical finite strain problems. It is, however, noticeable that the Qi6 element has shown superior results than the Q1E4 element in the Cook's membrane problem.

The presented results lead to the conclusion that the Qi5B-bar and Qi6B-bar are suitable to these problems, because they do not present any instabilities or any kind of locking.

The Qi5B-bar formulation is particularly attractive, since it has only two internal variables, corresponding to the bubble mode, and the results obtained in the Cook's membrane problem are nearly as good as the ones presented by the original Q1E4 element.

This formulation can be extended to the analysis of tridimensional problems, but in that case the selective integration can be advantageously replaced by an exact evaluation of the assumed dilatation, as discussed by Bonet and Bhargava [20].

\section{REFERENCES}

1. Nagtegaal JC, Parks DM, Rice JR. On numerically accurate finite element solutions in the fully plastic range. Computer Methods in Applied Mechanics and Engineering 1974; 4:153-177.

2. Pantuso D, Bathe K-J. On the stability of mixed finite elements in large strain analysis of incompressible solids. Finite Elements in Analysis and Design 1997; 28:83-104.

3. Simo JC, Armero F, Taylor RL. Improved versions of assumed enhanced strain tri-linear elements for 3D finite deformation problems. Computer Methods in Applied Mechanics and Engineering 1993; 110:359-386.

4. César de Sá JMA, Natal Jorge RM. New enhanced strain elements for incompressible problems. International Journal for Numerical Methods in Engineering 1999; 44:229-248.

5. Pian THH, Sumihara K. Rational approach for assumed stress finite elements. International Journal for Numerical Methods in Engineering 1984; 20:1685-1695.

6. Belytschko T, Bachrach WE. Efficient implementation of quadrilaterals with high coarse-mesh accuracy. Computer Methods in Applied Mechanics and Engineering 1986; 54:279-301.

7. Simo JC, Armero F. Geometrically non-linear enhanced strain mixed methods and the method of incompatible modes. International Journal for Numerical Methods in Engineering 1992; 33:1413-1449.

8. Lautersztajn N, Samuelsson A. Further discussion on four-node isoparametric quadrilateral elements in plane bending. International Journal for Numerical Methods in Engineering 2000; 47:129-140.

9. Brink U, Stein E. On some mixed finite element methods for incompressible and nearly incompressible finite elasticity. Computational Mechanics 1996; 19:105-119.

10. Miehe C. Aspects of the formulation and finite element implementation of large strain isotropic elasticity. International Journal for Numerical Methods in Engineering 1994; 37:1981-2004.

11. de Souza Neto EA, Peric D, Huang GC, Owen DRJ. Remarks on the stability of enhanced strain elements in finite elasticity and elastoplasticity. In Computational Plasticity: Fundamentals and Applications. Owen DRJ, Oñate E, Hinton E (eds), Proceedings of the Fourth International Conference, Barcelona, Spain, $1995 ; 1$. 
12. Glaser S, Armero F. On the formulation of enhanced strain finite elements in finite deformations. Engineering Computations 1997; 14(7):759-791.

13. Korelc J, Wriggers P. Consistent gradient formulation for a stable enhanced strain method for large deformation. Engineering Computations 1996; 13(1):103-123.

14. Wriggers P, Hueck U. A formulation of the qs6 element for large elastic deformations. International Journal for Numerical Methods in Engineering 1996; 39:1437-1454.

15. Korelc J, Wriggers P. Improved enhanced strain four-node element with Taylor expansion of the shape functions. International Journal for Numerical Methods in Engineering 1997; 40:407-421.

16. Hughes TJR. Generalization of selective integration procedures to anisotropic and nonlinear media. International Journal for Numerical Methods in Engineering 1980; 15(9):1413-1418.

17. Simo JC, Taylor RL, Pister KS. Variational and projection methods for the volume constraint in finite deformation elasto-plasticity. Computer Methods in Applied Mechanics and Engineering 1985; 51:177-208.

18. Moran B, Ortiz M, Shih CF. Formulation of implicit finite element methods for multiplicative finite deformation plasticity. International Journal for Numerical Methods in Engineering 1990; 29:483-514.

19. Zhu YY, Cescotto S. Unified and mixed formulation of the 4-node quadrilateral elements by assumed strain method: application to thermomechanical problems. International Journal for Numerical Methods in Engineering 1995; 38:685-716.

20. Bonet J, Bhargava P. A uniform deformation gradient hexahedron element with artificial hourglass control. International Journal for Numerical Methods in Engineering 1995; 38:2809-2828.

21. Reese S, Kussner M, Reddy BD. A new stabilization technique for finite elements in non-linear elasticity. International Journal for Numerical Methods in Engineering 1999; 44:1617-1652.

22. Belytschko T, Ong JS-J. Hourglass control in linear and nonlinear problems. Computer Methods in Applied Mechanics and Engineering 1984; 43:251-276.

23. Belytschko T, Bindeman LP. Assumed strain stabilization of the 4-node quadrilateral with 1-point quadrature for nonlinear problems. Computer Methods in Applied Mechanics and Engineering 1991; 88:311-340.

24. Bathe K-J. Finite Element Procedures. Prentice-Hall International Editions, 1996.

25. Crisfield MA, Norris V. A stabilised large-strain elasto-plastic Q1/P0 method. International Journal for Numerical Methods in Engineering 1999; 46:579-592.

26. Piltner R, Taylor RL. A systematic construction of B-bar functions for linear and non-linear mixed-enhanced finite elements for plane elasticity problems. International Journal for Numerical Methods in Engineering 1999; 44:615-639.

27. Taylor RL. Triangles, finite elements and mixed methods. ECCM'99 European Conference on Computational Mechanics, August 31-September 3 Munchen, Germany, 1999.

28. Armero F. On the locking and stability of finite elements in finite deformation plane strain problems. Computers and Structures 2000; 75:261-290.

29. César de Sá JMA, Owen DRJ. The imposition of the incompressibility constraint in finite elements-A review of methods with a new insight to the locking phenomenon. In Numerical Methods for Non-Linear Problems, Taylor C et al. (eds), Proceedings of the Third International Conference, Dubrovnik. Pineridge Press Ltd. Swansea, U.K., 1986.

30. César de Sá JMA. Numerical modeling of incompressible problems in glass forming and rubber technology. Ph.D. Thesis, University College of Swansea, 1986.

31. de Souza Neto EA, Peric D, Dutko M, Owen DRJ. Design of simple low order finite elements for large strain analysis of nearly incompressible solids. International Journal for Solids and Structures 1996; 33:3277-3296.

32. Simo JC. A framework for finite strain elastoplasticity based on maximum plastic dissipation and the multiplicative decomposition. part ii: computational aspects. Computer Methods in Applied Mechanics and Engineering 1988; 68:1-31.

33. Lubliner J. Plasticity Theory. Macmillan Publishing Company: New York, 1990.

34. César de Sá JMA, Areias PMA, Natal Jorge RM. Analysis of large elasto-plastic deformations with new compatible mode elements. ECCOMAS 2000, Barcelona, 11-14 September, 2000, accepted for publication.

35. Flanagan DP, Belytschko T. A uniform strain hexahedron and quadrilateral with orthogonal hourglass control. International Journal for Numerical Methods in Engineering 1981; 17:679-706.

36. Simo JC, Hughes TJR. Computational Inelasticity, Interdisciplinary Applied Mathematics, vol. 7. Springer: Berlin, 1998.

37. Simo JC. A framework for finite strain elastoplasticity based on maximum plastic dissipation and the multiplicative decomposition. part i: continuum formulation. Computer Methods in Applied Mechanics and Engineering 1988; 66:199-219.

38. Crisfield MA, Moita GF, Jelenic G, Lyons LPR. Enhanced lower-order element formulation for large strains. In Computational Plasticity: Fundamentals and Applications, Owen DRJ, Oñate E, Hinton E (eds), Proceedings of the Fourth International Conference, Barcelona, Spain, 1995; 1.

39. Shonauer M, de Souza Neto EA, Owen DRJ. Hencky tensor based enhanced large strain element for elastoplastic analysis. In Computational Plasticity: Fundamentals and Applications, Owen DRJ, Oñate E, Hinton E (eds), Proceedings of the Fourth International Conference, Barcelona, Spain, 1995; 1. 